\title{
Synthesis of Novel Organosilicon Compounds Possessing Fully Substituted Imidazole Nucleus Sonocatalyzed by Cu-Fe/ZSM-5 Bimetallic Oxides
}

Kazem D. Safa, ${ }^{* a}$ Aynaz Feyzi, ${ }^{a}$ Maryam Allahvirdinesbat, ${ }^{a}$ Leila Sarchami, ${ }^{a}$ Parvaneh Nakhostin Panahi ${ }^{b}$

${ }^{a}$ Organosilicon Research Laboratory, Faculty of Chemistry, University of Tabriz, 5166616471 Tabriz, Iran

${ }^{b}$ Department of Chemistry, Faculty of Science, University of Zanjan, Iran

Corresponding author. email: dsafa@tabrizu.ac.ir

\section{SUPPLEMENTARY MATERIALS}

General procedure for preparation of 1,2,4,5-tetrasubstituted imidazoles

In a $50 \mathrm{~mL}$ round bottom flask benzil $(1 \mathrm{mmol})$, aldehyde $(1 \mathrm{mmol})$, amine $(1 \mathrm{mmol})$, and ammonium acetate $(1.1$ mmol) were taken in the presence of $3 \mathrm{wt} . \%$ of Fe-Cu/ZSM-5 in $10 \mathrm{~mL}$ of ethanol under ultrasonic irradiation. After completion of the reaction (monitored by TLC, within $2-5 \mathrm{~min}$ ), the crude residue was cooled to room temperature. Then catalyst was separated by filtration. The filtrate was evaporated to give the crude product. This product was washed with n-hexane and purified by recrystallization from ethanol. The supported reagent was washed thrice with ethanol and dried under vacuum before reuse.

Spectral data of some representative products are given below:

4-(2,4,5-triphenyl-1H-imidazol-1-yl)phenol (1a)

White powder (99\%), M.P. $>280{ }^{\circ} \mathrm{C}$, IR $\left(\mathrm{KBr}, \mathrm{cm}^{-1}\right): v_{\max }=3414,3054,2922,1604,1513,1446,696 .{ }^{1} \mathrm{H}$ NMR $\left(\right.$ DMSO-d $\left._{6}, 400 \mathrm{MHz}\right) \delta_{\mathrm{H}}: 6.66(\mathrm{~d}, J=8.5 \mathrm{~Hz}, 2 \mathrm{H}, \mathrm{Ar}-\mathrm{H}), 7.04(\mathrm{~d}, J=8.5 \mathrm{~Hz}, 2 \mathrm{H}, \mathrm{Ar}-\mathrm{H}), 7.14-7.30(\mathrm{~m}, 11 \mathrm{H}, \mathrm{Ar}-\mathrm{H})$, 7.42-7.44 (m, 2H, Ar-H), 7.49 (d, J 7.5, 2H, Ar-H), 9.78 (s, 1H, Ar-OH). (DMSO-d 6 , 100 MHz) $\delta_{\mathrm{C}}: 115.59,126.35$, $127.84,128.12,128.15,128.27,128.40,129.79,130.58,130.63,131.12,131.59,134.56,136.61,146.11,157.31$. Anal.Calc. for $\mathrm{C}_{27} \mathrm{H}_{20} \mathrm{~N}_{2} \mathrm{O}$ : C, 83.48; H, 5.19; N, 7.21\%. Found: C, 83.54; H, 5.07; N, 7.19\%. 


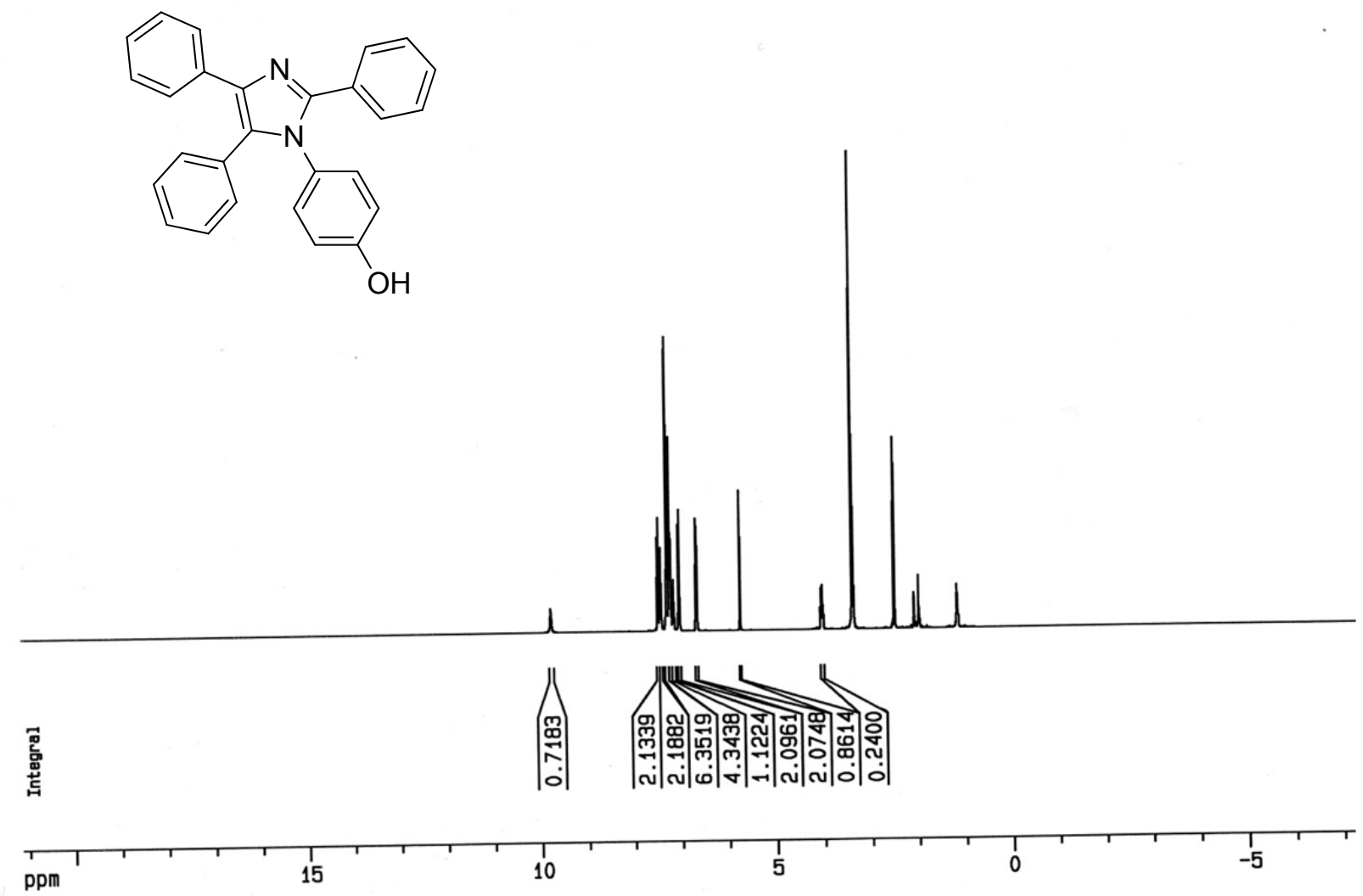

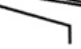

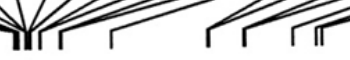

${ }^{1} \mathrm{H}$ NMR spectrum of 4-(2,4,5-triphenyl-1H-imidazol-1-yl)phenol (1a) in DMSO-d $\mathrm{d}_{6}$ 


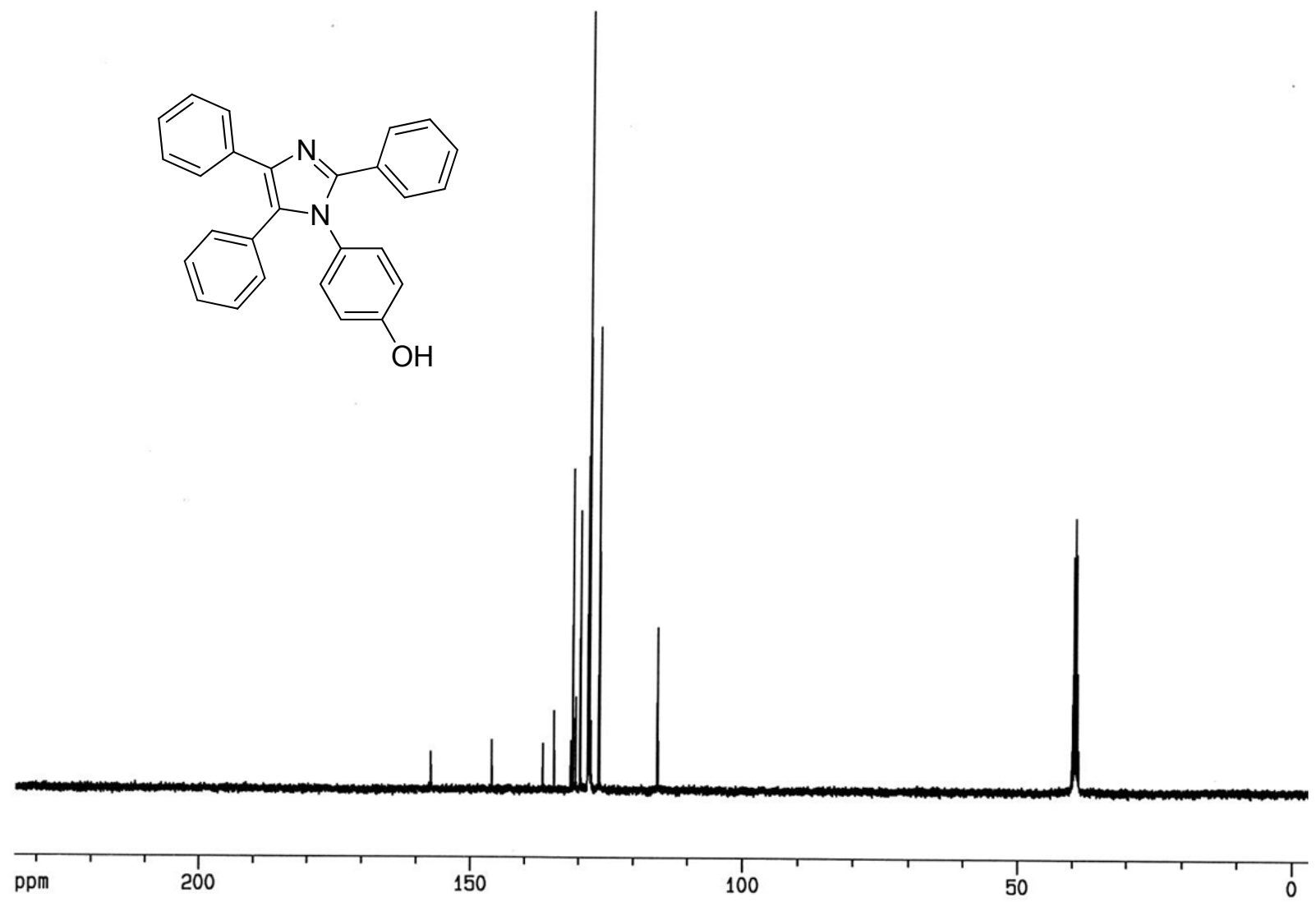

${ }^{13}$ C NMR spectrum of 4-(2,4,5-triphenyl-1H-imidazol-1-yl)phenol (1a) in DMSO-d 6 


\section{4-(2-(4-chlorophenyl)-4,5-diphenyl-1H-imidazol-1-yl)phenol (1c)}

White powder (99\%), M.P. $>280^{\circ} \mathrm{C}$, IR $\left(\mathrm{KBr}, \mathrm{cm}^{-1}\right): v_{\max }=3416,3054,2926,1595,1513,1478,1440,745,695$.

${ }^{1} \mathrm{H}$ NMR (DMSO-d 6 , $\left.400 \mathrm{MHz}\right) \delta_{\mathrm{H}}: 6.67$ (d, $\left.J=8.4 \mathrm{~Hz}, 2 \mathrm{H}, \mathrm{Ar}-\mathrm{H}\right), 7.05$ (d, $\left.J=8.4 \mathrm{~Hz}, 2 \mathrm{H}, \mathrm{Ar}-\mathrm{H}\right), 7.14-7.43$ (m, 12H, Ar-H), 7.47 (d, J = 7.5 Hz, 2H, Ar-H), 9.82 (s, 1H, Ar-OH). (DMSO-d 6 , 100 MHz) $\delta_{\mathrm{C}}: 115.69,126.33,126.46$, $127.55,128.15,128.27,128.37,128.43,129.37,129.71,129.74,130.44,131.10,131.89,132.98,134.37,136.76$, 144.96, 157.42. Anal.Calc. for $\mathrm{C}_{27} \mathrm{H}_{19} \mathrm{ClN}_{2} \mathrm{O}$ : C, 76.68; H, 4.53; N, 6.62\%. Found: C, 76.49; H, 4.61; N, 6.58\%.

言
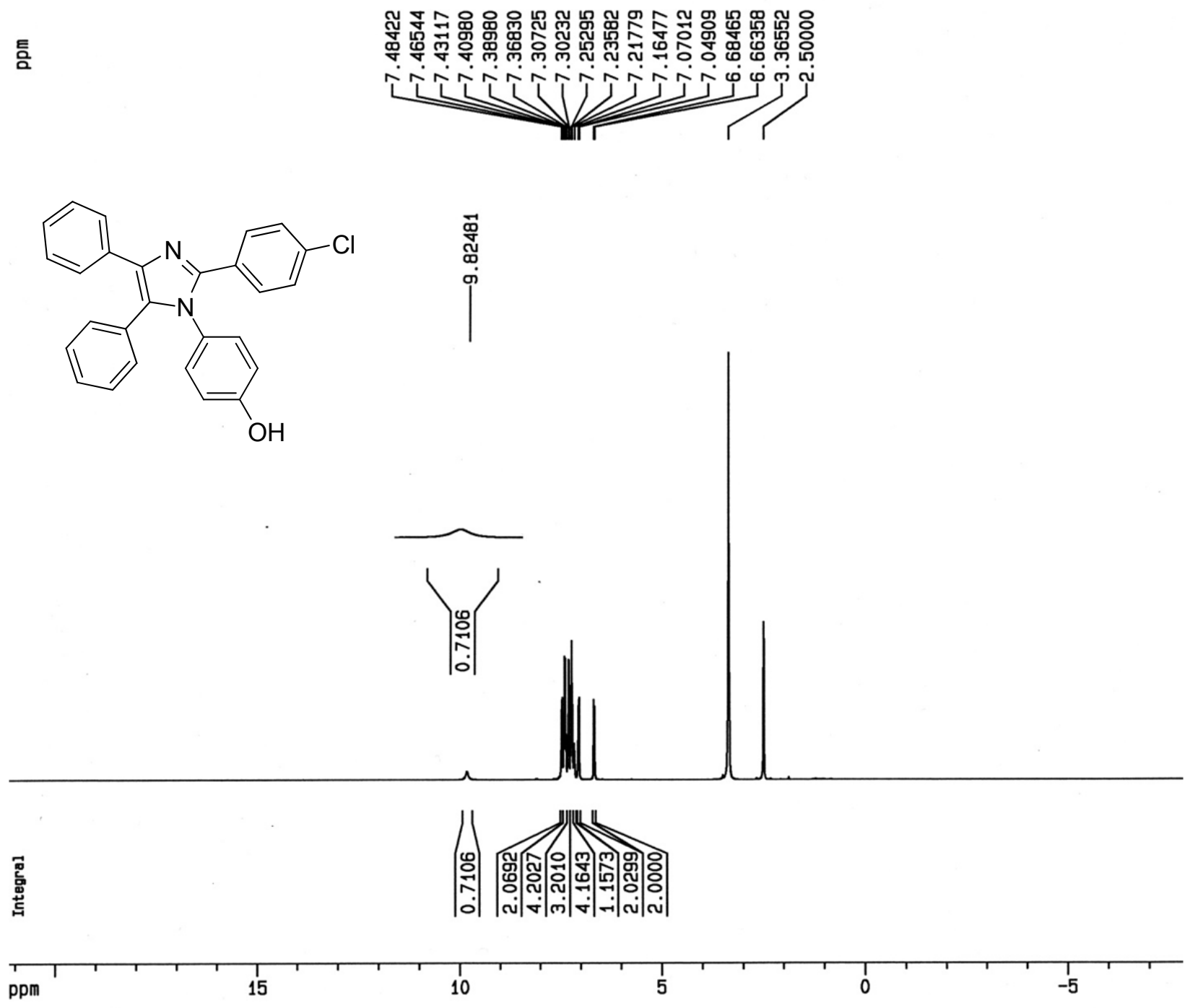

${ }^{1} \mathrm{H}$ NMR spectrum of 4-(2-(4-chlorophenyl)-4,5-diphenyl-1H-imidazol-1-yl)phenol (1c) in DMSO-d 6 


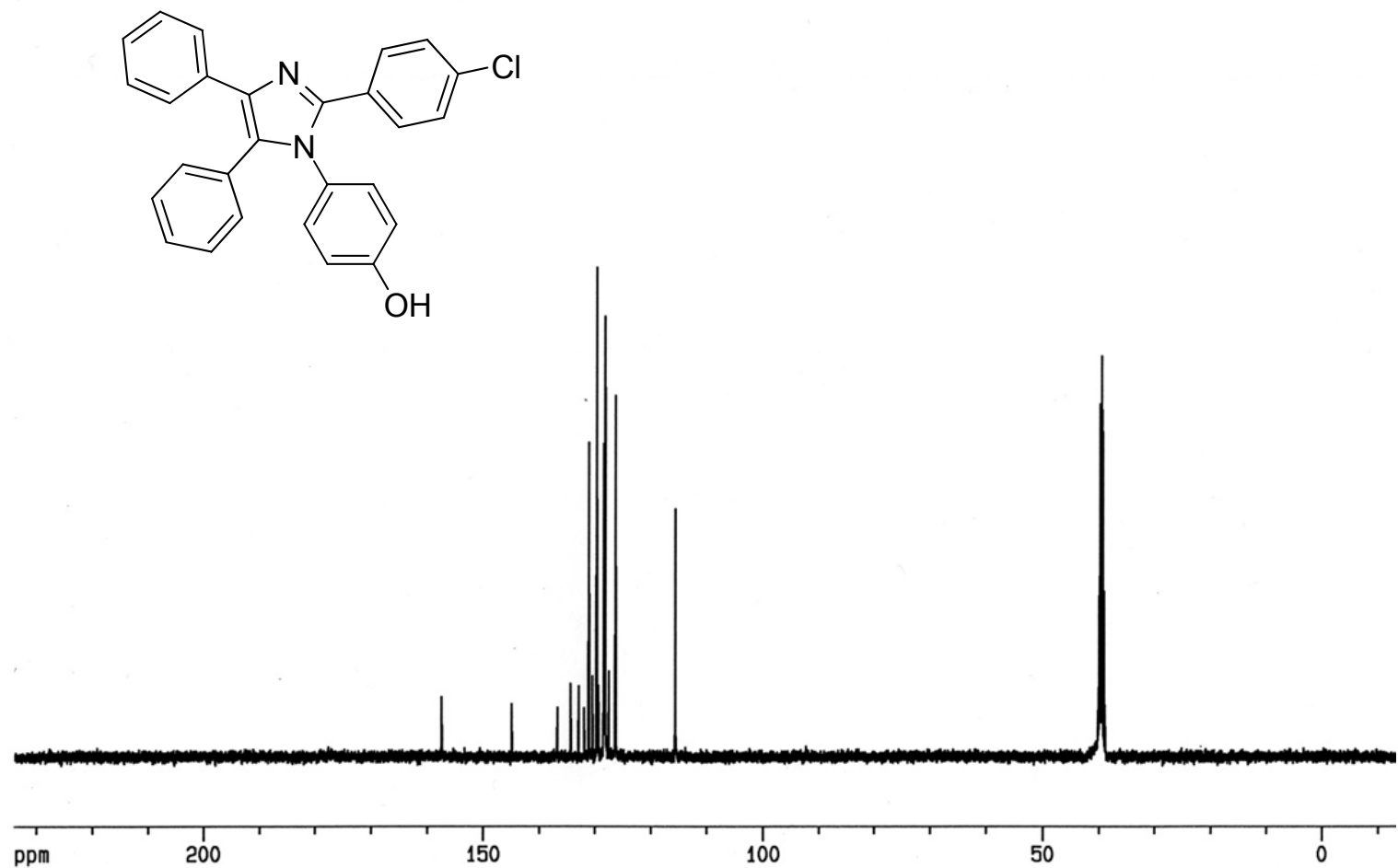

${ }^{13} \mathrm{C}$ NMR spectrum of 4-(2-(4-chlorophenyl)-4,5-diphenyl-1H-imidazol-1-yl)phenol (1c) in DMSO-d 6 


\section{4,4'-(4,5-diphenyl-1H-imidazole-1,2-diyl)diphenol (1d)}

White powder (97\%), M.P. $>280{ }^{\circ} \mathrm{C}$, IR $\left(\mathrm{KBr}, \mathrm{cm}^{-1}\right): v_{\max }=3416,3053,2924,1642,1513,1438,694 .{ }^{1} \mathrm{H}$ NMR $\left(\right.$ DMSO-d $_{6}, 400$ MHz) $\delta_{\mathrm{H}}:$ 6.64-6.67 (m, 4H, Ar-H), 7.00 (d, J = 8.4, 2H, Ar-H), 7.12-7.29 (m, 10H, Ar-H), 7.45 (d, $J=7.7,2 \mathrm{H}, \mathrm{Ar}-\mathrm{H}), 9.73$ (bs, 2H, Ar-OH). (DMSO-d $\left.d_{6}, 100 \mathrm{MHz}\right) \delta_{\mathrm{C}}: 114.92,115.55,121.48,126.22,126.31$, $128.05,128.09,128.16,128.39,129.67,129.84,130.86,130.89,131.14,134.75,136.15,146.56,157.19,157.52$. Anal.Calc. for $\mathrm{C}_{27} \mathrm{H}_{20} \mathrm{~N}_{2} \mathrm{O}_{2}$ : C, 80.18; H, 4.98; N, 6.93\%. Found: C, 80.01; H, 4.75; N, 6.87\%. 
틈
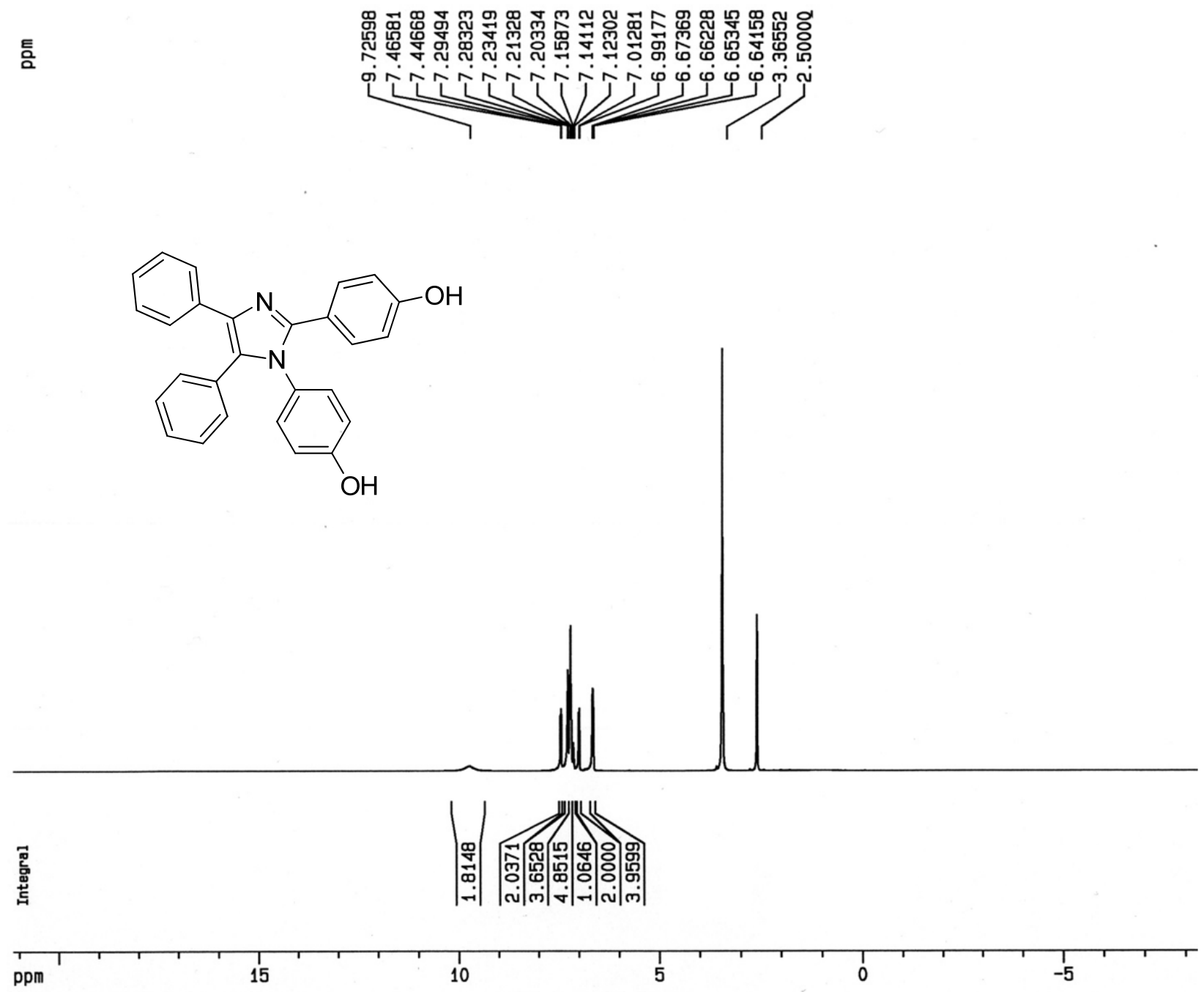

${ }^{1} \mathrm{H}$ NMR spectrum of 4,4'-(4,5-diphenyl-1H-imidazole-1,2-diyl)diphenol (1d) in DMSO-d 6 


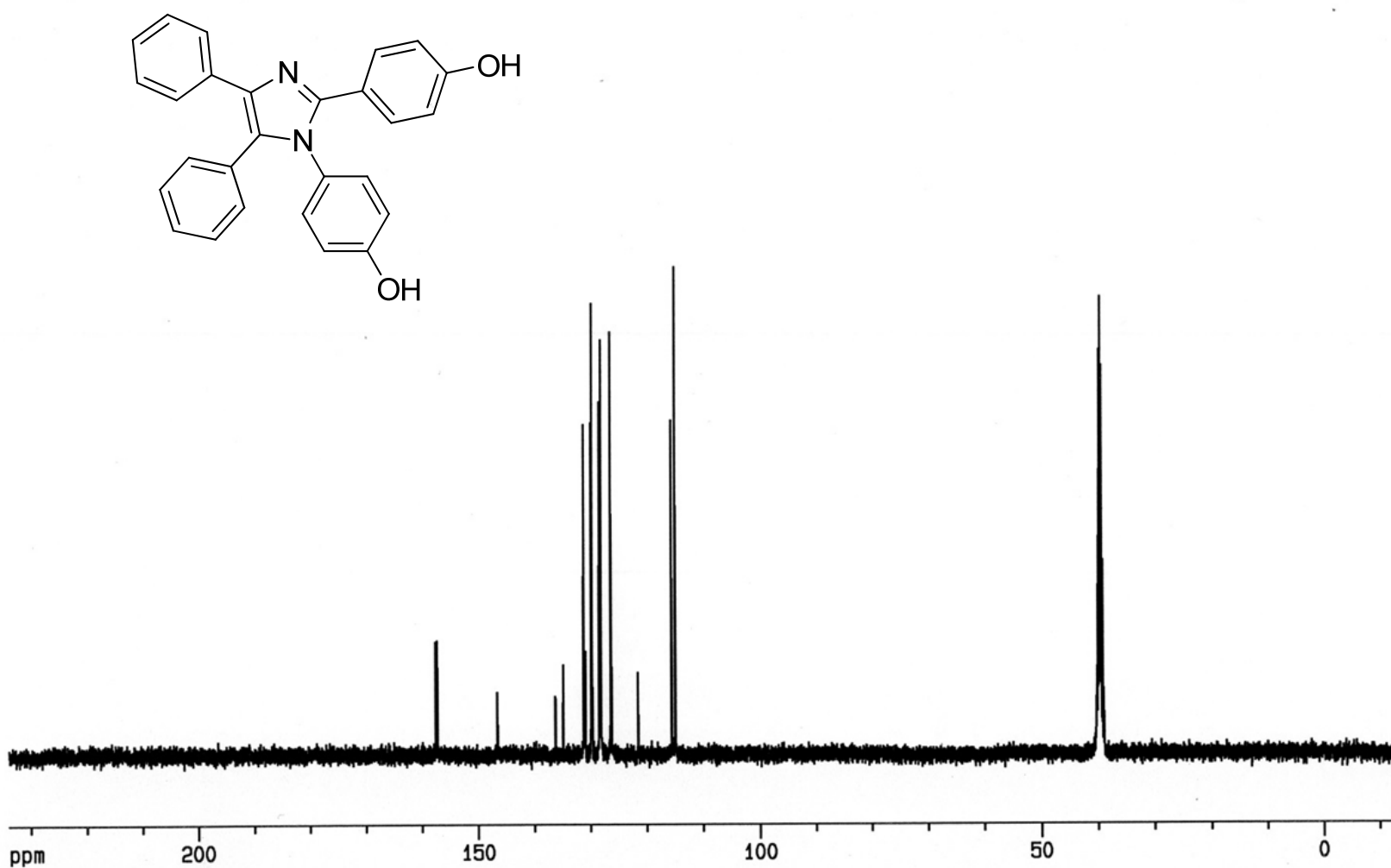

${ }^{13} \mathrm{C}$ NMR spectrum of 4,4'-(4,5-diphenyl-1H-imidazole-1,2-diyl)diphenol (1d) in DMSO-d 6 


\section{2-(4-Chlorophenyl)-4,5-diphenyl-1-p-tolyl-1H-imidazole (1f)}

White powder (97\%), M.P. $=167-169^{\circ} \mathrm{C}$, IR $\left(\mathrm{KBr}, \mathrm{cm}^{-1}\right): v_{\max }=3054,2976,1510,1478,1445,745,696 .{ }^{1} \mathrm{H}$ NMR $\left(\mathrm{CDCl}_{3}, 400 \mathrm{MHz}\right): \delta_{\mathrm{H}} 2.36\left(\mathrm{~s}, 3 \mathrm{H}, \mathrm{CH}_{3}\right), 6.95(\mathrm{~d}, J=8.1 \mathrm{~Hz}, 2 \mathrm{H}, \mathrm{Ar}-\mathrm{H}), 7.10(\mathrm{~d}, J=8.1 \mathrm{~Hz}, 2 \mathrm{H}, \mathrm{Ar}-\mathrm{H}), 7.16-7.18$ (m, 2H, Ar-H), 7.21-7.30 (m, 8H, Ar-H), 7.41-7.43 (m, 2H, Ar-H), 7.61-7.63 (m, 2H, Ar-H). ${ }^{13} \mathrm{C}$ NMR $\left(\mathrm{CDCl}_{3}, 100\right.$ MHz): $\delta_{\mathrm{C}} 20.14\left(\mathrm{CH}_{3}\right), 119.77,125.61,126.30,126.95,126.99,127.13,127.29,127.31,128.10,128.82,129.03$, 129.50, 130.05, 130.17, 133.15, 133.21, 133.31, 137.41, 144.74. Anal.Calc. for $\mathrm{C}_{28} \mathrm{H}_{21} \mathrm{ClN}_{2}$ : C, 79.89; $\mathrm{H}, 5.03 ; \mathrm{N}$, 6.66\%. Found: C, 79.76; H, 5.14; N, 6.86\%. 
틈
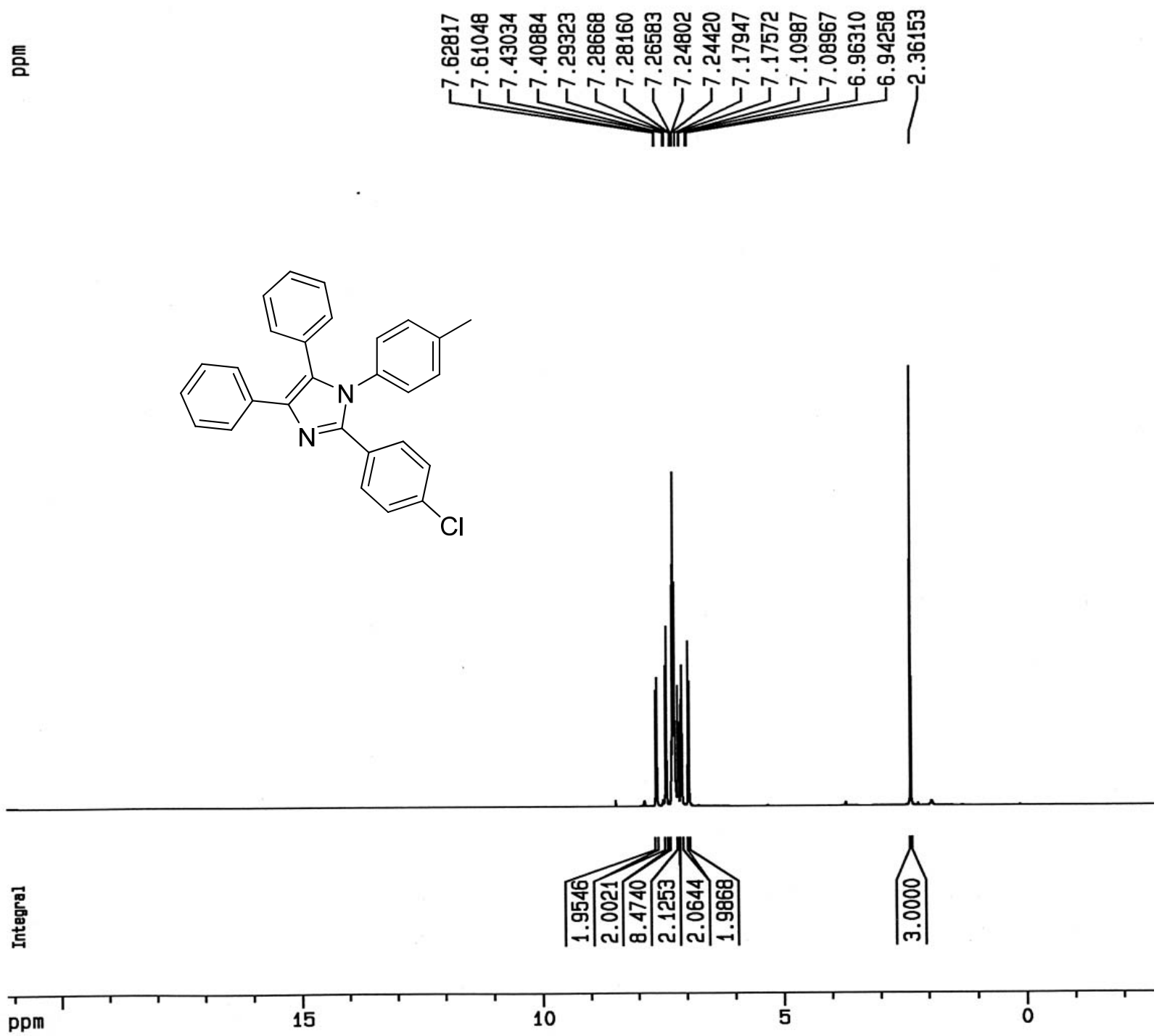

${ }^{1} \mathrm{H}$ NMR spectrum of 2-(4-Chlorophenyl)-4,5-diphenyl-1-p-tolyl-1H-imidazole (1f) in $\mathrm{CDCl}_{3}$ 

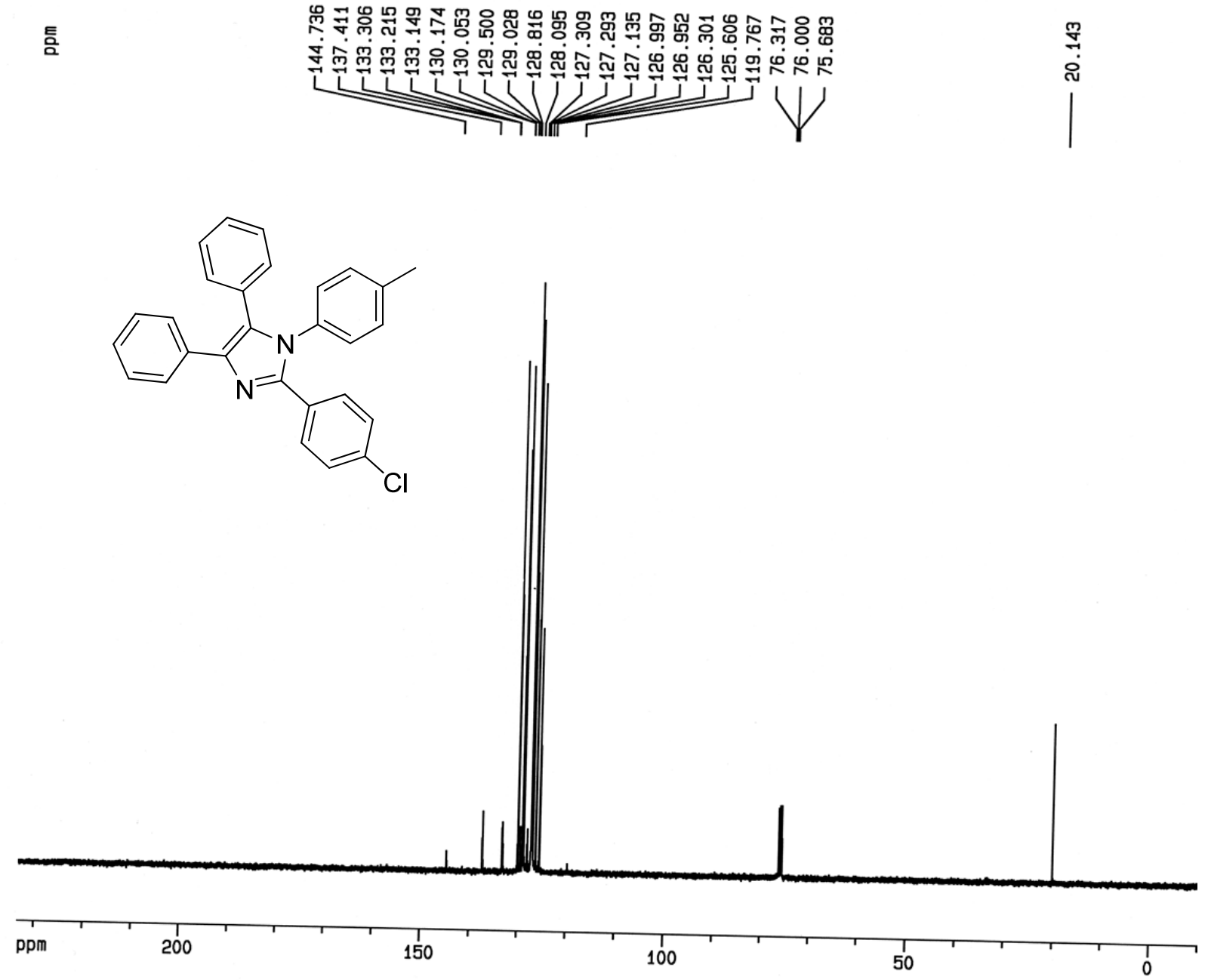

${ }^{13} \mathrm{C}$ NMR spectrum of 2-(4-Chlorophenyl)-4,5-diphenyl-1-p-tolyl- $1 \mathrm{H}$-imidazole (1f) in $\mathrm{CDCl}_{3}$ 


\section{1-(4-Bromophenyl)-4,5-diphenyl-2-p-tolyl-1H-imidazole $1 \mathrm{~g}$}

White powder (98\%), M.P. $=190-192{ }^{\circ} \mathrm{C}, \mathrm{IR}\left(\mathrm{KBr}, \mathrm{cm}^{-1}\right): v_{\max }=3445,3057,2961,2908,1570,1510,1478,726$, 697. ${ }^{1} \mathrm{H}$ NMR $\left(\mathrm{CDCl}_{3}, 400 \mathrm{MHz}\right): \delta_{\mathrm{H}} 2.36\left(\mathrm{~s}, 3 \mathrm{H}, \mathrm{CH}_{3}\right), 6.93$ (d, J = 8.6 Hz, 2H, Ar-H), 7.11-7.17 (m, 4H, Ar-H), 7.20-7.35 (m, 8H, Ar-H), 7.41 (d, 2H, $J=8.6 \mathrm{~Hz}, \mathrm{Ar}-\mathrm{H}), 7.60-7.62(\mathrm{~m}, 2 \mathrm{H}, \mathrm{Ar}-\mathrm{H}) .{ }^{13} \mathrm{C}$ NMR $\left(\mathrm{CDCl}_{3}, 100 \mathrm{MHz}\right): \delta_{\mathrm{C}}$ $20.27\left(\mathrm{CH}_{3}\right), 121.06,121.58,125.64,126.37,127.14,127.51,127.88,127.96,128.56,128.91,129.35,129.44$, 130.10, 131.13, 131.25, 133.27, 135.27, 137.42, 146.04. Anal.Calc. for $\mathrm{C}_{28} \mathrm{H}_{21} \mathrm{BrN}_{2}$ : C, 72.26; H, 4.55; N, 6.02\%. Found: C, 72.14; H, 4.47; N, 6.14\%. 
镸
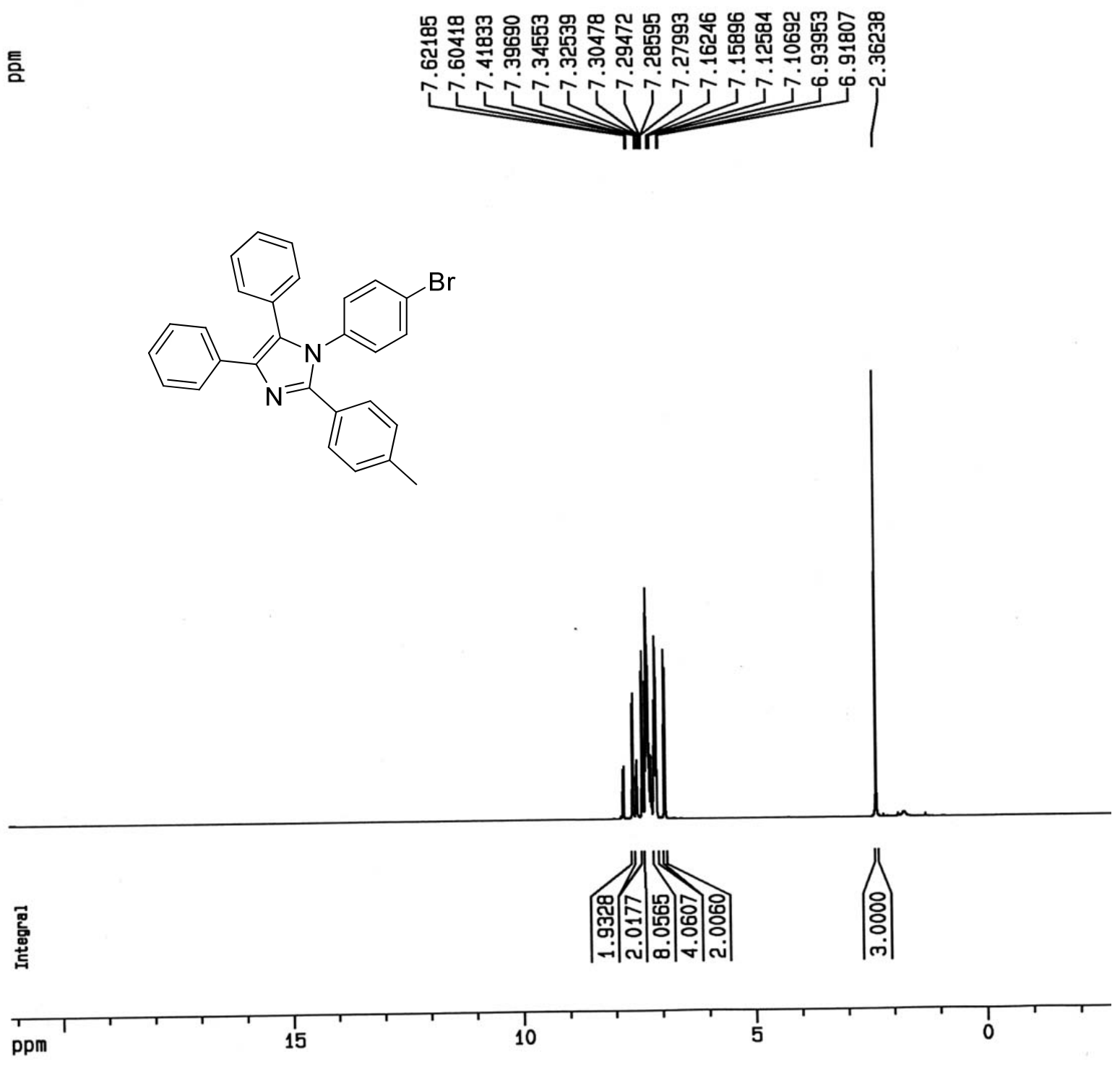

${ }^{1} \mathrm{H}$ NMR spectrum of 1-(4-Bromophenyl)-4,5-diphenyl-2-p-tolyl-1H-imidazole (1g) in $\mathrm{CDCl}_{3}$ 


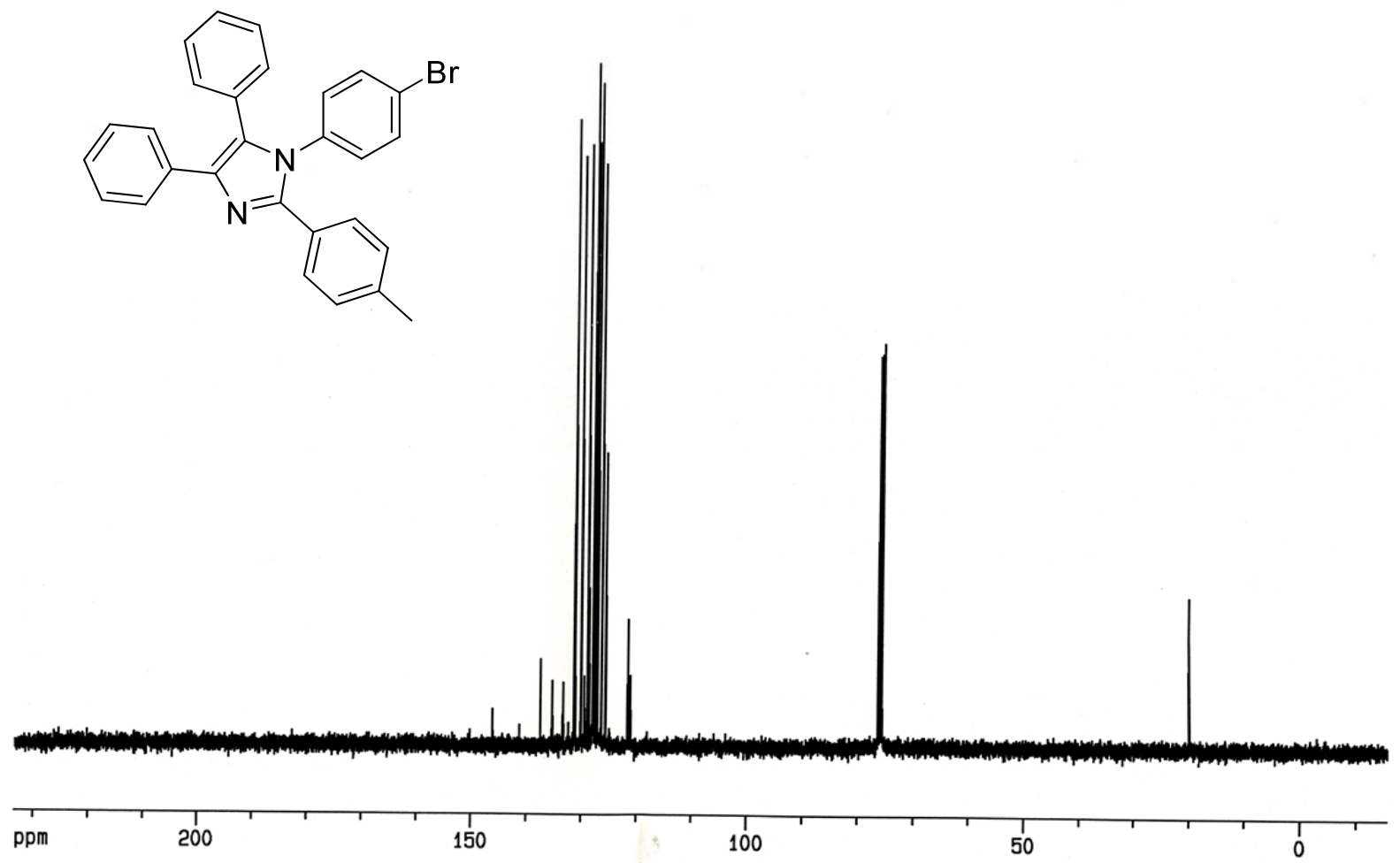

${ }^{13} \mathrm{C}$ NMR spectrum of 1-(4-Bromophenyl)-4,5-diphenyl-2-p-tolyl-1 $\mathrm{H}$-imidazole (1g) in $\mathrm{CDCl}_{3}$ 


\section{1-(3-Chlorophenyl)-4,5-diphenyl-2-p-tolyl-1H-imidazole (1i)}

White powder (99\%), M.P. $=198-200^{\circ} \mathrm{C}$, IR $\left(\mathrm{KBr}, \mathrm{cm}^{-1}\right): v_{\max }=3056,2963,2909,1490,1479,739,703 .{ }^{1} \mathrm{H}$ NMR $\left(\mathrm{CDCl}_{3}, 400 \mathrm{MHz}\right): \delta_{\mathrm{H}} 2.33\left(\mathrm{~s}, 3 \mathrm{H}, \mathrm{CH}_{3}\right), 6.93-6.95(\mathrm{~m}, 1 \mathrm{H}, \mathrm{Ar}-\mathrm{H}), 7.05(\mathrm{t}, J=1.86 \mathrm{~Hz}, 1 \mathrm{H}, \mathrm{ArH}), 7.08(\mathrm{~d}, 2 \mathrm{H}, J=$ 8.04 Hz, Ar-H), 7.12-7.27 (m, 10H, Ar-H), 7.32 (d, 2H, $J=8.15 \mathrm{~Hz}, \mathrm{Ar}-\mathrm{H}), 7.58-7.60$ (m, 2H, Ar-H). ${ }^{13} \mathrm{C}$ NMR $\left(\mathrm{CDCl}_{3}, 100 \mathrm{MHz}\right): \delta_{\mathrm{C}} 20.27\left(\mathrm{CH}_{3}\right), 125.64,125.77,126.24,126.33,127.14,127.15,127.47,127.58,127.78$, 127.95, 128.92, 129.31, 130.05, 133.21, 133.48, 137.33, 137.45, 146.00. Anal.Calc. for $\mathrm{C}_{28} \mathrm{H}_{21} \mathrm{ClN}_{2}$ : C, 79.89; $\mathrm{H}$, $5.03 ;$ N, 6.66\%. Found: C, 79.78; H, 5.12; N, 6.74\%. 


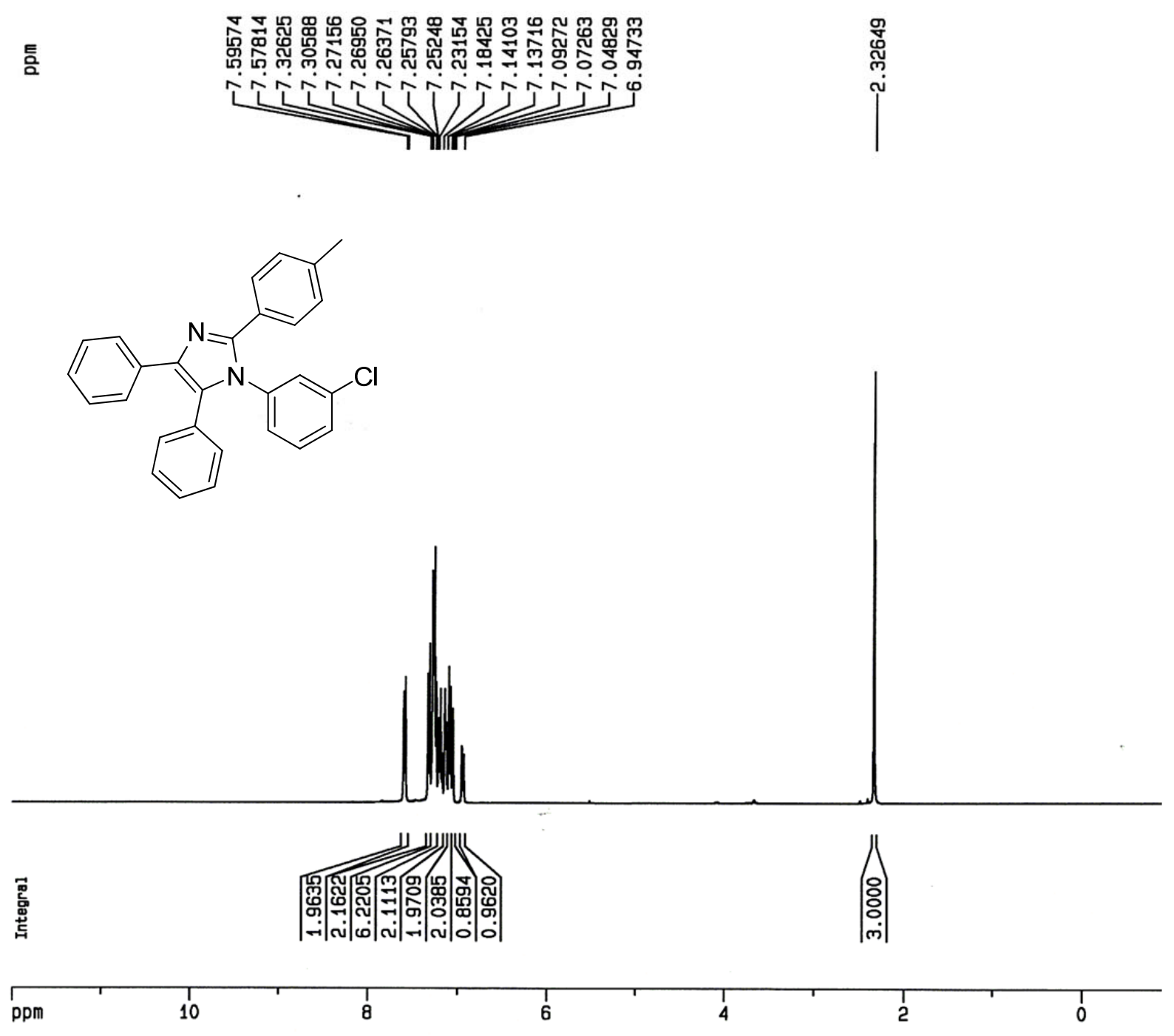

${ }^{1} \mathrm{H}$ NMR spectrum of 1-(3-Chlorophenyl)-4,5-diphenyl-2-p-tolyl-1H-imidazole (1i) in CDCl3 


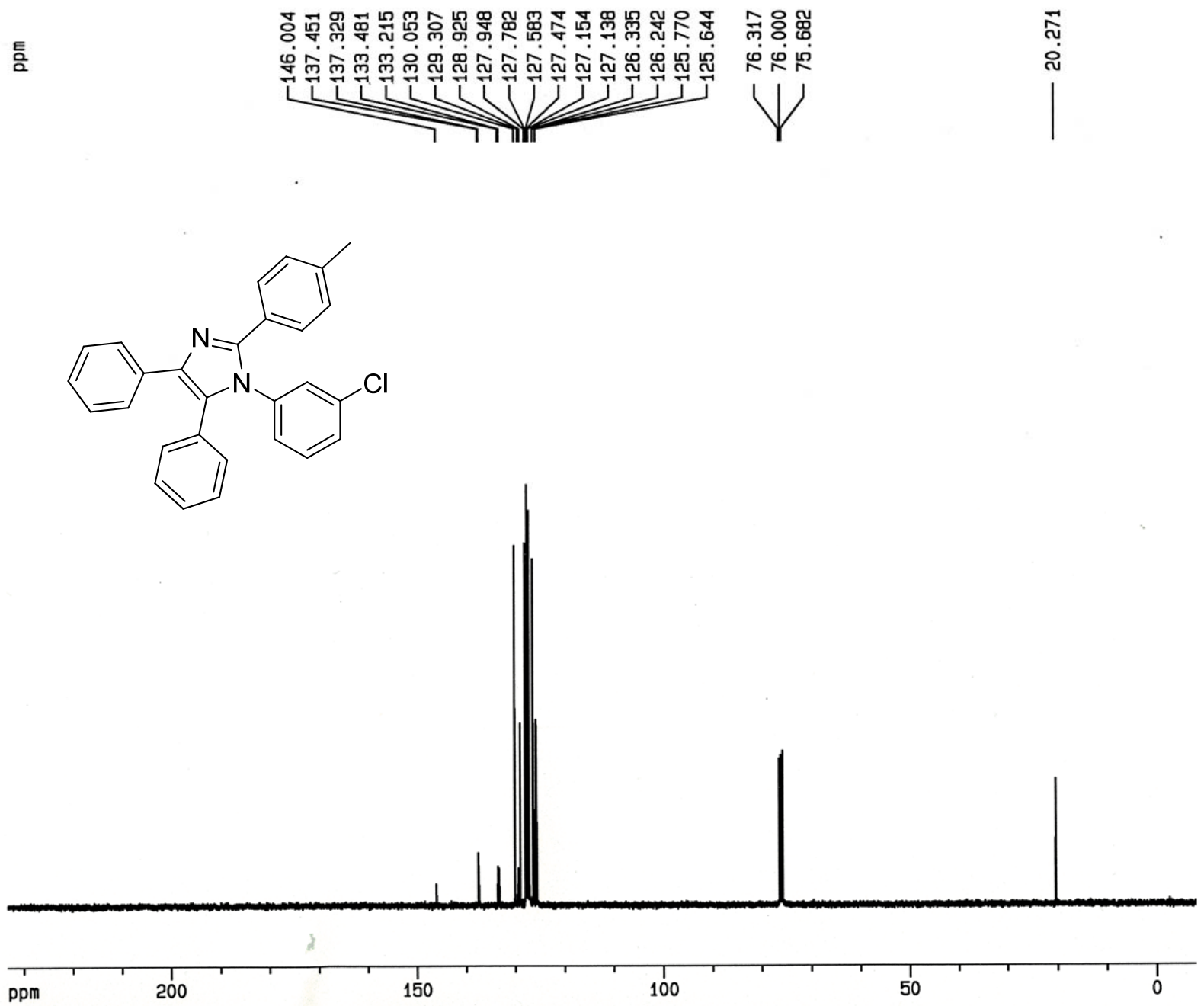

${ }^{13} \mathrm{C}$ NMR spectrum of 1-(3-Chlorophenyl)-4,5-diphenyl-2-p-tolyl-1H-imidazole (1i) in CDCl3 


\section{1-(4-Fluorophenyl)-4,5-diphenyl-2-p-tolyl-1H-imidazole (11)}

White powder (97\%), M.P. $=188-190{ }^{\circ} \mathrm{C}, \mathrm{IR}\left(\mathrm{KBr}, \mathrm{cm}^{-1}\right): v_{\max }=3058,2962,2908,1602,1508,1444,960,775$, 730. ${ }^{1} \mathrm{H}$ NMR $\left(\mathrm{CDCl}_{3}, 400 \mathrm{MHz}\right): \delta_{\mathrm{H}} 2.36\left(\mathrm{~s}, 3 \mathrm{H}, \mathrm{CH}_{3}\right), 6.96-7.00(\mathrm{~m}, 2 \mathrm{H}, \mathrm{Ar}-\mathrm{H}), 7.03-7.07(\mathrm{~m}, 2 \mathrm{H}, \mathrm{Ar}-\mathrm{H}), 7.11(\mathrm{~d}$, $2 \mathrm{H}, J=8.0 \mathrm{~Hz}, \mathrm{Ar}-\mathrm{H}), 7.14-7.17$ (m, 2H, Ar-H), 7.21-7.32 (m, 6H, Ar-H), 7.35 (d, 2H, J=8.0 Hz, Ar-H), 7.63 (d, $2 \mathrm{H}, J=7.2 \mathrm{~Hz}, \mathrm{Ar}-\mathrm{H}),{ }^{13} \mathrm{C} \mathrm{NMR}\left(\mathrm{CDCl}_{3}, 100 \mathrm{MHz}\right): \delta_{\mathrm{C}} 20.24\left(\mathrm{CH}_{3}\right), 114.95,115.17,125.58,126.32,126.41$, $127.01,127.12,127.41,127.79,127.88,128.99,129.08,129.50,129.58,130.07,132.19,133.30,137.16,137.31$, 146.12, 159.59, 162.06. Anal.Calc. for $\mathrm{C}_{28} \mathrm{H}_{21} \mathrm{FN}_{2}$ : C, 83.14; H, 5.23; N, 6.93\%. Found: C, 83.09; H, 5.14; N, $6.81 \%$. 

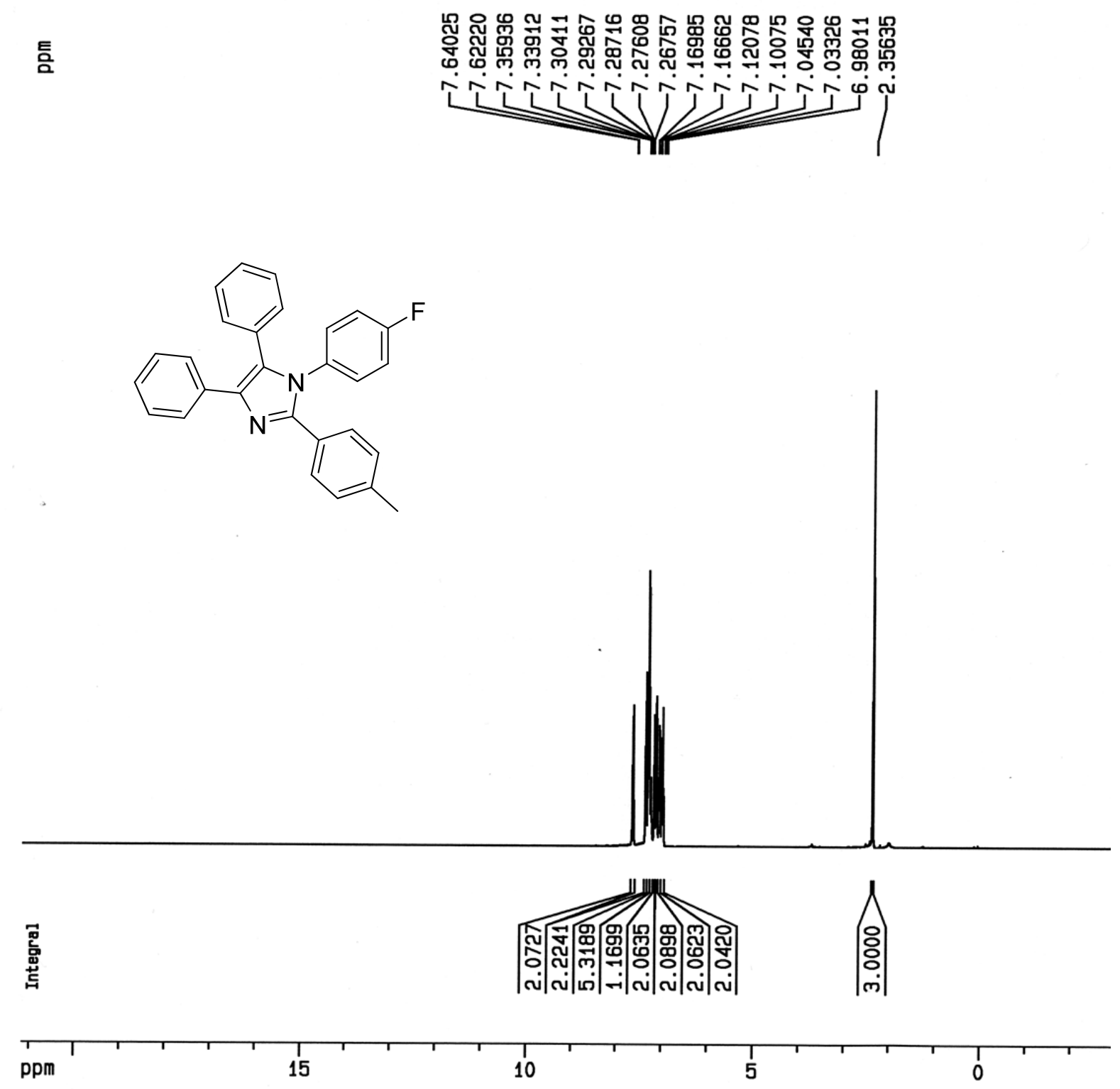

${ }^{1} \mathrm{H}$ NMR spectrum of 1-(4-Fluorophenyl)-4,5-diphenyl-2-p-tolyl-1 $H$-imidazole (11) in CDCl3 

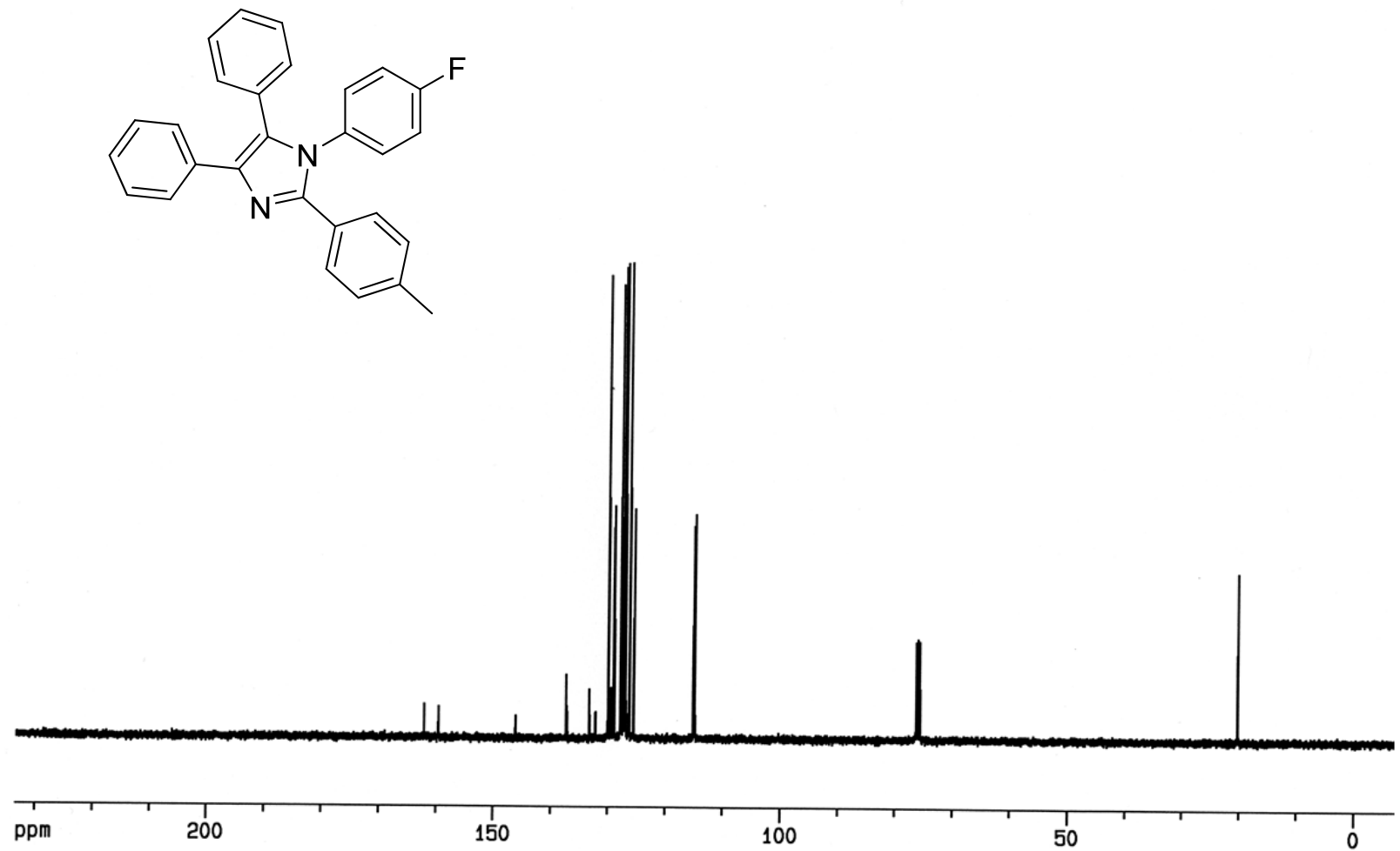

${ }^{13} \mathrm{C}$ NMR spectrum of 1-(4-Fluorophenyl)-4,5-diphenyl-2-p-tolyl-1H-imidazole (11) in CDCl3 


\section{General procedure for bromination of imidazole derivatives}

A mixture of (1 mmol) imidazole derivative (1a or 1c) and (1 mmol) dibromoalkane (1,2-dibromoethane, 1,3dibromopropane or 1,4-dibromobutane ), also (1 mmol) imidazole derivative (1d) and (1 mmol) 1,4-dibromobutane in present of $\mathrm{K}_{2} \mathrm{CO}_{3}$ in $100 \mathrm{ml}$ of dry DMF was stirred at room temperature for $24 \mathrm{~h}$. Then DMF solvent was evaporated in vacuum, and the residue was taken up in $\mathrm{CH}_{2} \mathrm{Cl}_{2}(50 \mathrm{ml})$ and the extract was washed with brine (30 $\mathrm{ml}$ ) and then dried $\left(\mathrm{Na}_{2} \mathrm{SO}_{4}\right)$. After filtration the solvent evaporated, and the residue was purified by silica gel column chromatography using hexane/ethylacetate $(75: 25, \mathrm{v} / \mathrm{v})$. Fraction containing 4-bromoalkoxy-imidazole was collected then the solvent was removed under reduced pressure.

\section{Spectral data of some novel representative products are given below:}

\section{1-(4-(4-bromobutoxy)phenyl)-2,4,5-triphenyl-1H-imidazole (2a)}

Yellow powder $(69 \%)$, M.P. $=132-134{ }^{\circ} \mathrm{C}, \mathrm{IR}\left(\mathrm{KBr}, \mathrm{cm}^{-1}\right): v_{\max }=3058,2924,1609,1510,1469,1165,1101,695$.

${ }^{1} \mathrm{H}$ NMR $\left(\mathrm{CDCl}_{3}, 400 \mathrm{MHz}\right): \delta_{\mathrm{H}} 1.90-1.95\left(\mathrm{~m}, 2 \mathrm{H}, \mathrm{CH}_{2}\right), 2.01-2.08\left(\mathrm{~m}, 2 \mathrm{H}, \mathrm{CH}_{2}\right), 3.48\left(\mathrm{t}, J 6.5,2 \mathrm{H}, \mathrm{CH}_{2}\right), 3.94(\mathrm{t}, J$ 6.0, 2H, $\left.\mathrm{CH}_{2}\right), 6.73$ (d, J 8.8, 2H, Ar-H), 6.94 (d, J 8.8, 2H, Ar-H), 7.12-7.15 (m, 2H, Ar-H), 7.16-7.26 (m, 9H, Ar$\mathrm{H}), 7.43-7.46(\mathrm{~m}, 2 \mathrm{H}, \mathrm{Ar}-\mathrm{H}), 7.58$ (d, $J=7.3,2 \mathrm{H}, \mathrm{Ar}-\mathrm{H}) .{ }^{13} \mathrm{C} \mathrm{NMR}\left(\mathrm{CDCl}_{3}, 100 \mathrm{MHz}\right): \delta_{\mathrm{C}} 28.3,28,67,32.30,65.96$ $\left(\mathrm{CH}_{2}\right), 113.61,125.49,126.34,126.85,127.07,127.09,127.31,127.89,127.88,128.15,128.42,128.90,129.62$, 129.76, 130.12, 133.50, 146.00, 157.37. Anal.Calc. for $\mathrm{C}_{31} \mathrm{H}_{27} \mathrm{BrN}_{2} \mathrm{O}$ : C, 71.13; H, 5.20; N, 5.35\%. Found: C, 71.33; H, 5.38; N, 5.21\%. 
틈
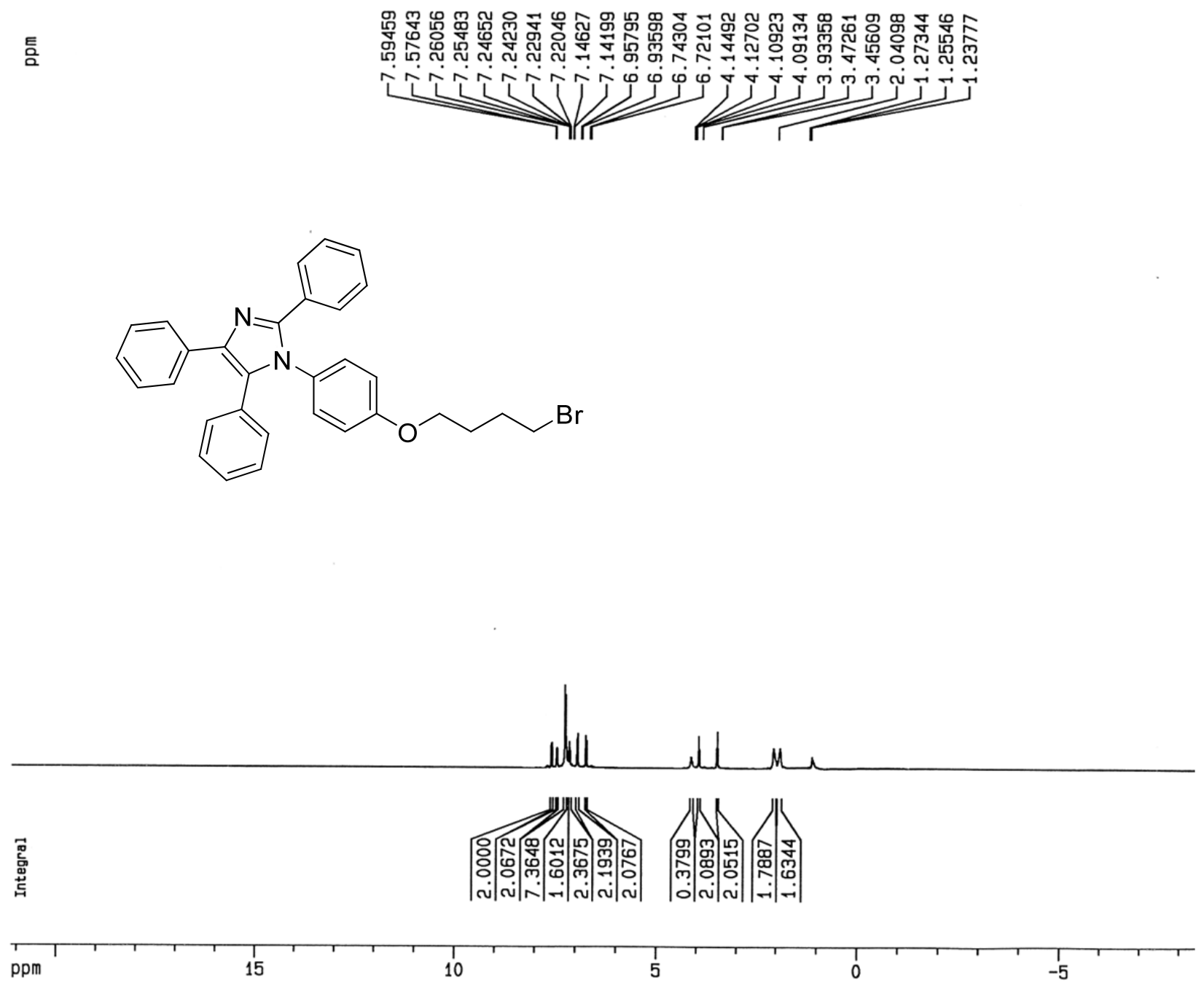

${ }^{1} \mathrm{H}$ NMR spectrum of 1-(4-(4-bromobutoxy)phenyl)-2,4,5-triphenyl-1 $\mathrm{H}$-imidazole (2a) in $\mathrm{CDCl}_{3}$ 


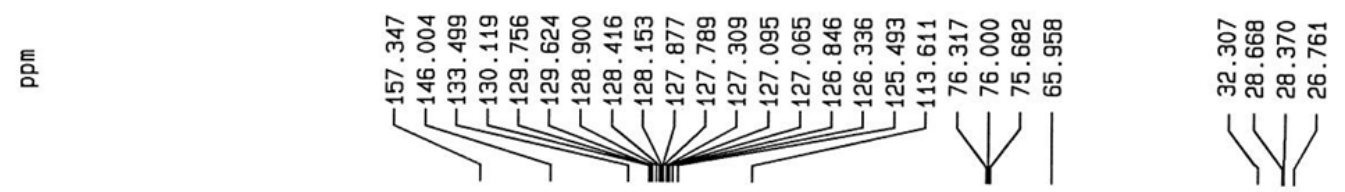<smiles>BrCCCCOc1ccc(-n2c(-c3ccccc3)nc(-c3ccccc3)c2-c2ccccc2)cc1</smiles>

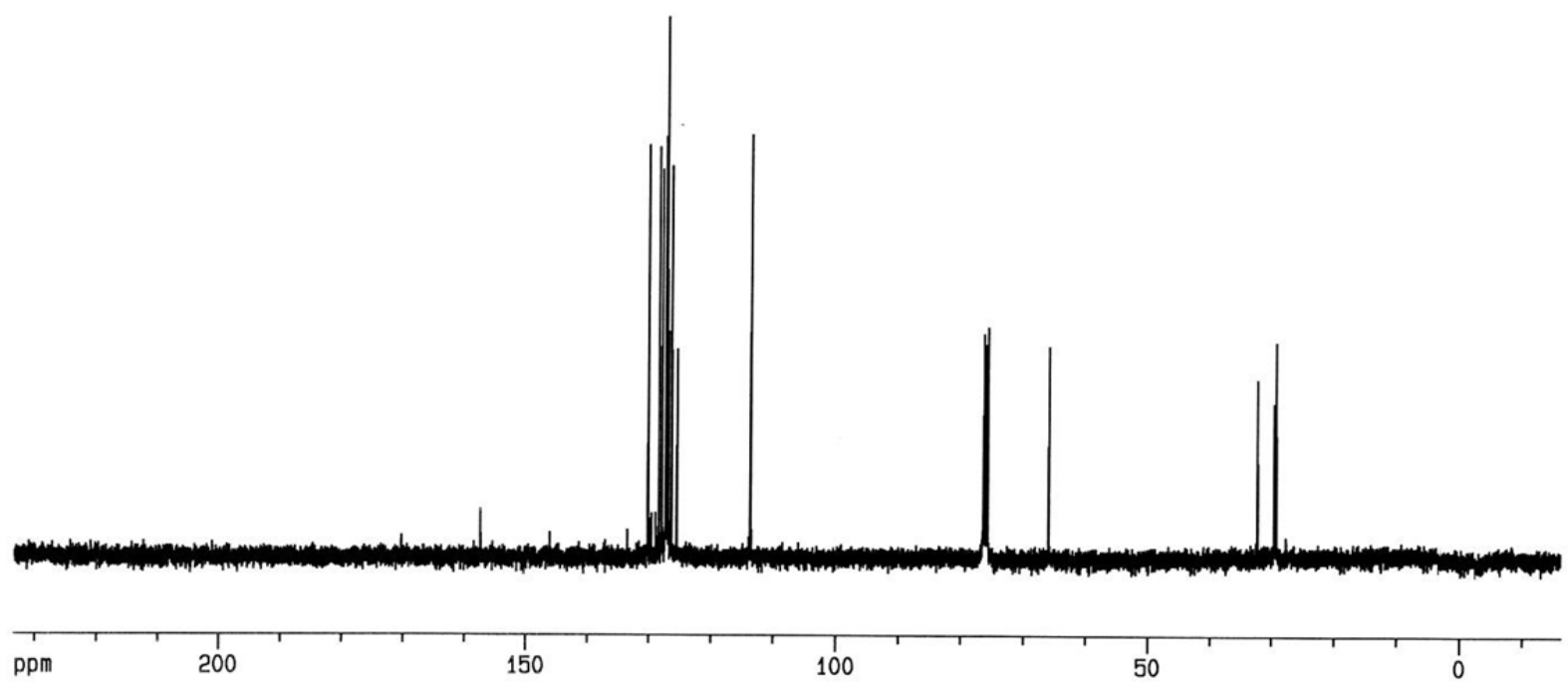

${ }^{13} \mathrm{C}$ NMR spectrum of 1-(4-(4-bromobutoxy)phenyl)-2,4,5-triphenyl- $1 \mathrm{H}$-imidazole (2a) in $\mathrm{CDCl}_{3}$ 


\section{2-(4-(2-bromoethoxy)phenyl)-4,5-diphenyl-1-p-tolyl-1H-imidazole (2b)}

White powder $(60 \%)$, M.P. $=164-166^{\circ} \mathrm{C}, \mathrm{IR}\left(\mathrm{KBr}, \mathrm{cm}^{-1}\right)=3057,2924,1617,1539,1512,1457,1130,615 .{ }^{1} \mathrm{H}$ NMR $\left(\mathrm{CDCl}_{3}, 400 \mathrm{MHz}\right): \delta_{\mathrm{H}} 2.32\left(\mathrm{~s}, 3 \mathrm{H}, \mathrm{CH}_{3}\right), 3.62\left(\mathrm{t}, J=6.3 \mathrm{~Hz}, 2 \mathrm{H}, \mathrm{CH}_{2}\right), 4.26\left(\mathrm{t}, J=6.3 \mathrm{~Hz}, 2 \mathrm{H}, \mathrm{CH}_{2}\right), 6.78(\mathrm{~d}$, $J=8.8 \mathrm{~Hz}, 2 \mathrm{H}, \mathrm{Ar}-\mathrm{H}), 6.91(\mathrm{~d}, J=8.2 \mathrm{~Hz}, 2 \mathrm{H}, \mathrm{Ar}-\mathrm{H}), 7.05(\mathrm{~d}, J=8.2 \mathrm{~Hz}, 2 \mathrm{H}, \mathrm{Ar}-\mathrm{H}), 7.11-7.26(\mathrm{~m}, 8 \mathrm{H}, \mathrm{Ar}-\mathrm{H}), 7.37$ (d, $J=7.0 \mathrm{~Hz}, 2 \mathrm{H}, \mathrm{Ar}-\mathrm{H}), 7.58(\mathrm{~d}, J=7.2 \mathrm{~Hz}, 2 \mathrm{H}, \mathrm{Ar}-\mathrm{H}) .{ }^{13} \mathrm{C} \mathrm{NMR}\left(\mathrm{CDCl}_{3}, 100 \mathrm{MHz}\right): \delta_{\mathrm{C}} 20.16\left(\mathrm{CH}_{3}\right), 27.94$, $66.68\left(\mathrm{CH}_{2}\right), 113.24,123.02,125.47,126.36,126.80,127.11,127.12,127.27,128.69,129.37,129.61,129.80$, 130.12, 133.52, 137.11, 145.68, 156.96. Anal.Calc. for $\mathrm{C}_{30} \mathrm{H}_{25} \mathrm{BrN}{ }_{2} \mathrm{O}: \mathrm{C}, 70.73 ; \mathrm{H}, 4.95 ; \mathrm{N}, 5.50 \%$. Found: C, 70.63; $\mathrm{H}, 4.86 ; \mathrm{N}, 5.41 \%$. 


\section{镸}

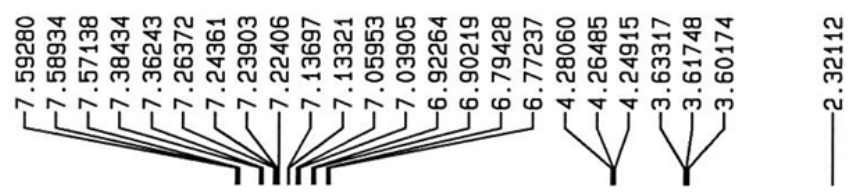<smiles>Cc1ccc(-n2c(-c3ccc(OCCBr)cc3)nc(-c3ccccc3)c2-c2ccccc2)cc1</smiles>
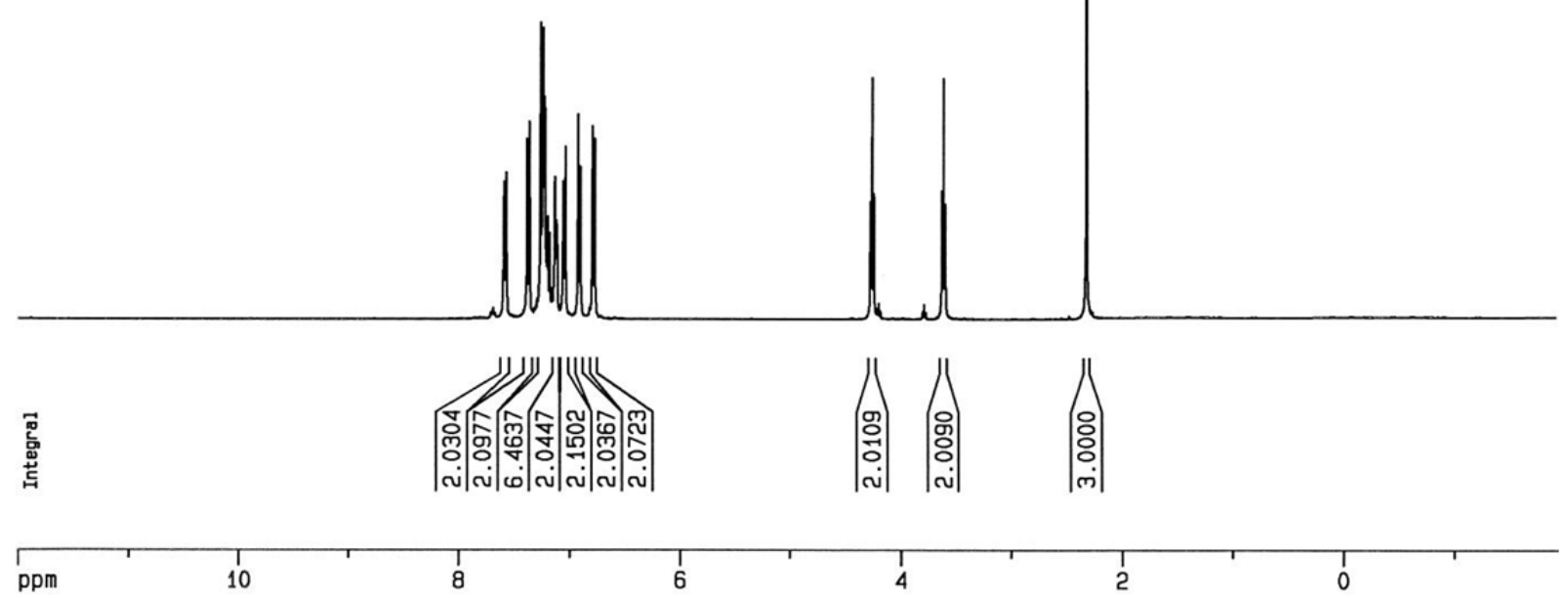

${ }^{1} \mathrm{H}$ NMR spectrum of 2-(4-(2-bromoethoxy)phenyl)-4,5-diphenyl-1-p-tolyl- $1 \mathrm{H}$-imidazole (2b) in $\mathrm{CDCl}_{3}$ 
<smiles>Cc1ccc(-n2c(-c3ccccc3)nc(-c3ccccc3)c2-c2ccc(OCCBr)cc2)cc1</smiles>
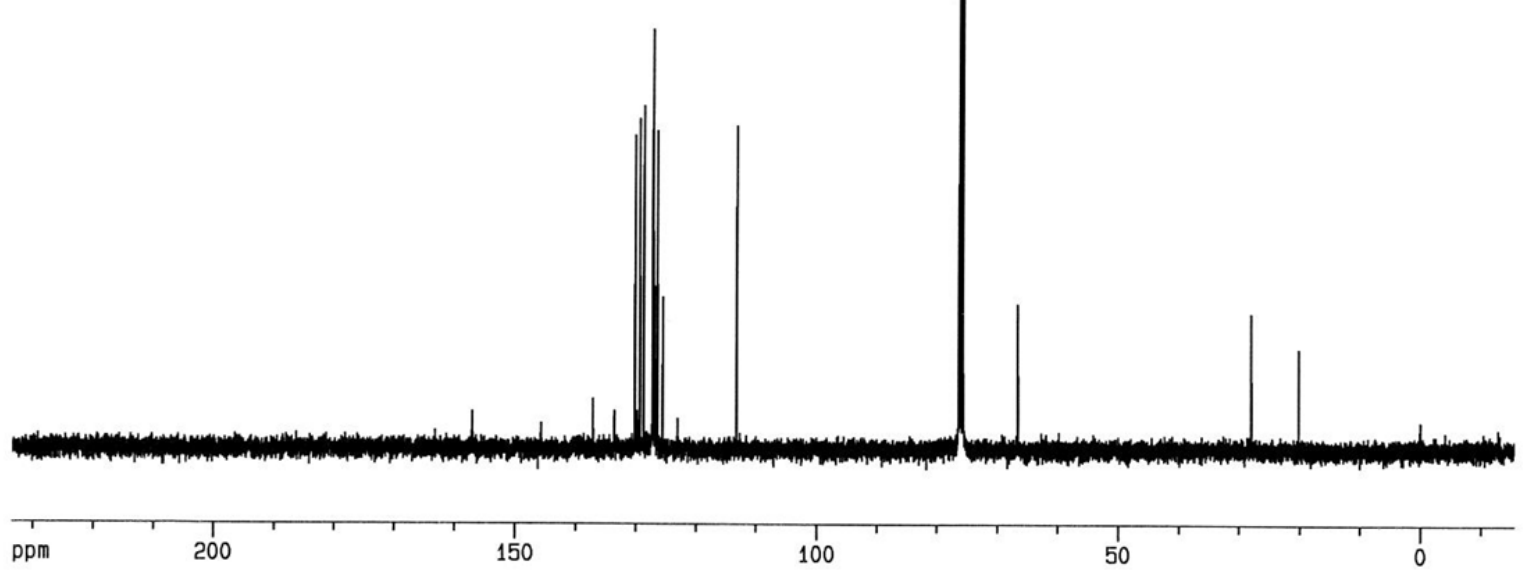

${ }^{13} \mathrm{C}$ NMR spectrum of 2-(4-(2-bromoethoxy)phenyl)-4,5-diphenyl-1-p-tolyl-1 $\mathrm{H}$-imidazole (2b) in $\mathrm{CDCl}_{3}$ 


\section{1-(4-(4-bromobutoxy) phenyl)-2-(4-chlorophenyl)-4,5-diphenyl-1H-imidazole (2c)}

White powder $(64 \%)$, M.P. $=148-150^{\circ} \mathrm{C}$, IR $\left(\mathrm{KBr}, \mathrm{cm}^{-1}\right)=3057,2927,1650,1605,1562,1541,1511,1475,1417$, 1244, 1167, 1091, 1014, 777, 754, 725, 695, 657, 542. ${ }^{1} \mathrm{H}$ NMR ( $\left.\mathrm{CDCl}_{3}, 400 \mathrm{MHz}\right): \delta_{\mathrm{H}} 1.90-1.96\left(\mathrm{~m}, 2 \mathrm{H}, \mathrm{CH}_{2}\right)$, 2.02-2.09 (m, 2H, $\left.\mathrm{CH}_{2}\right), 3.48\left(\mathrm{t}, J=6.5 \mathrm{~Hz}, 2 \mathrm{H}, \mathrm{CH}_{2}\right), 3.94$ (t, $\left.J=6.0 \mathrm{~Hz}, 2 \mathrm{H}, \mathrm{CH}_{2}\right), 6.72-6.76(\mathrm{~m}, 2 \mathrm{H}, \mathrm{Ar}-\mathrm{H}), 6.91-$ 6.95 (m, 2H, Ar-H).7.11-7.14 (m, 2H, Ar-H), 7.16-7.26 (m, 8H, Ar-H), 7.36-7.39 (m, 2H, Ar-H), 7.55-7.57 (m, 2H,

Ar-H). ${ }^{13} \mathrm{C} \mathrm{NMR}\left(\mathrm{CDCl}_{3}, 100 \mathrm{MHz}\right): \delta_{\mathrm{C}} 26.74,28.35,32.37,65.97\left(\mathrm{CH}_{2}\right), 113.73,125.54,125.63,126.29,126.97$, $127.16,127.34,127.36,128.05,128.34,128.57,129.01,129.47,130.05,130.30,133.16,133.28,137.24,144.84$, 157.49. Anal.Calc. for $\mathrm{C}_{31} \mathrm{H}_{26} \mathrm{BrClN}_{2} \mathrm{O}$ : C, 66.74; H, 4.70; N, 5.02\%. Found: C, 66.63; H, 4.88; N, 5.31\%. 
镸

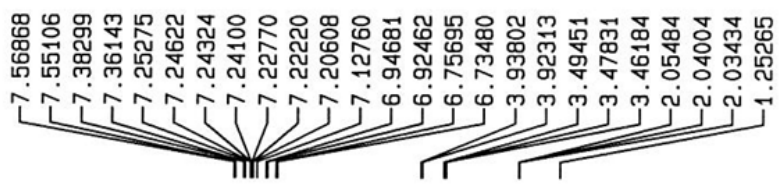<smiles>Clc1ccc(-c2nc(-c3ccccc3)c(-c3ccccc3)n2-c2ccc(OCCCCBr)cc2)cc1</smiles>
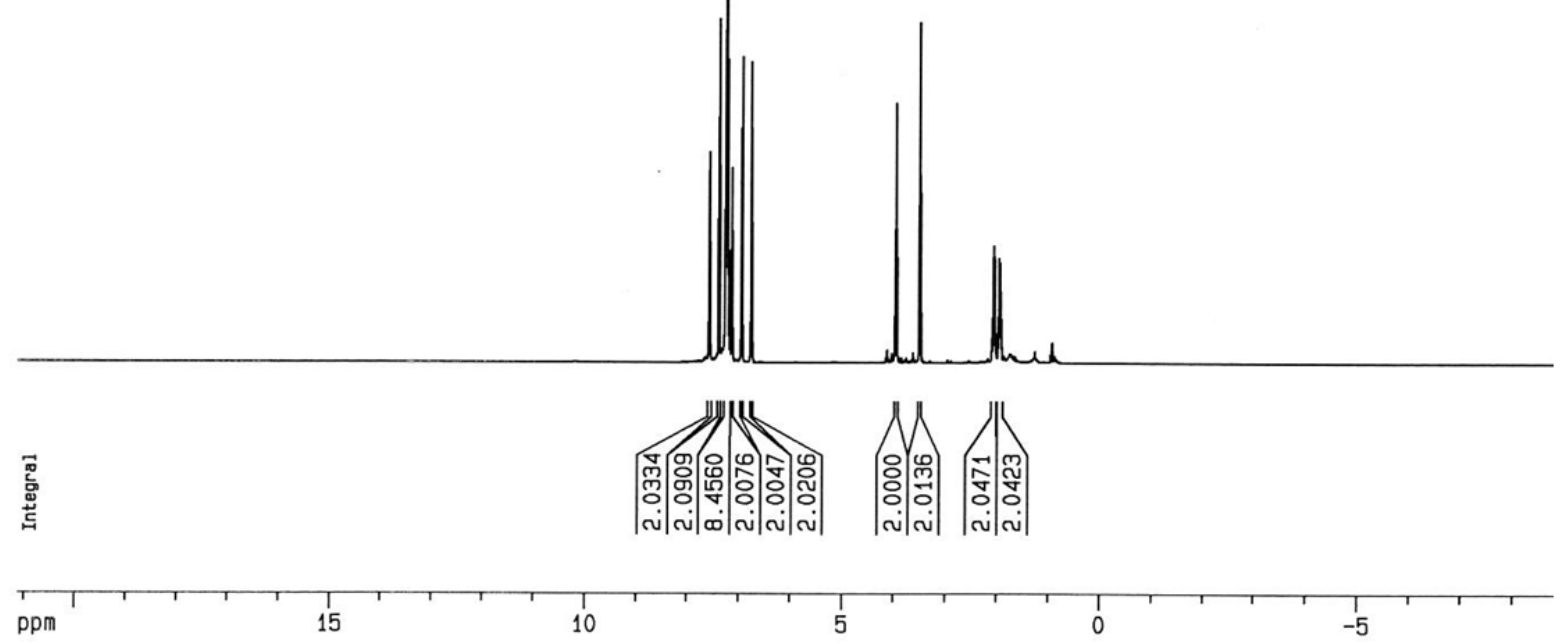

${ }^{1} \mathrm{H}$ NMR spectrum of 1-(4-(4-bromobutoxy) phenyl)-2-(4-chlorophenyl)-4,5-diphenyl-1H-imidazole (2c) in $\mathrm{CDCl}_{3}$ 


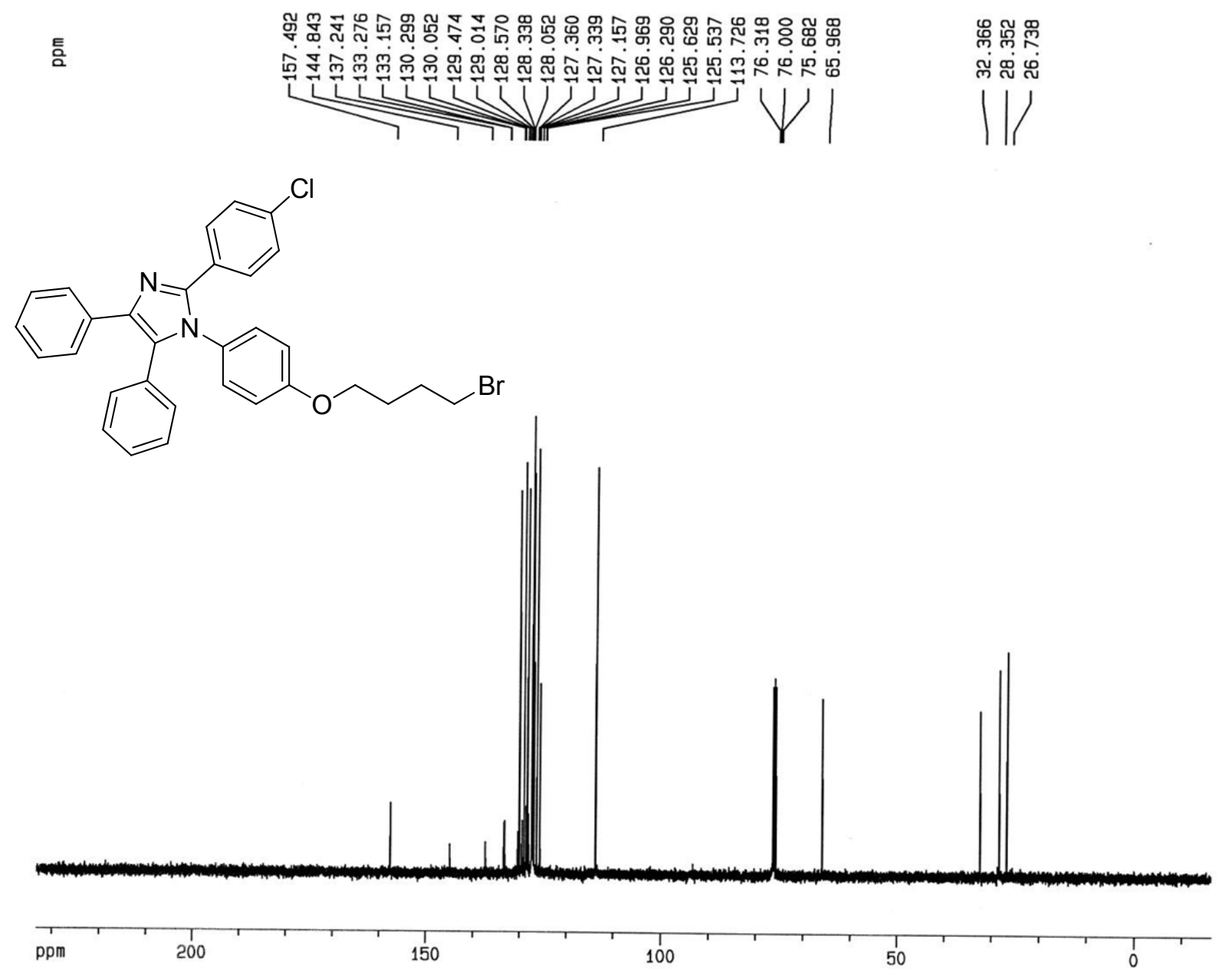

${ }^{13} \mathrm{C}$ NMR spectrum of 1-(4-(4-bromobutoxy) phenyl)-2-(4-chlorophenyl)-4,5-diphenyl-1H-imidazole (2c) in $\mathrm{CDCl}_{3}$ 


\section{1,2-bis(4-(4-bromobutoxy)phenyl)-4,5-diphenyl-1H-imidazole (2d)}

White powder $(59 \%)$, M.P. $=171-173^{\circ} \mathrm{C}, \mathrm{IR}\left(\mathrm{KBr}, \mathrm{cm}^{-1}\right)=3057,2924,1616,1539,1512,1462,1109,1028,695$.

${ }^{1} \mathrm{H}$ NMR (CDCl3, $\left.400 \mathrm{MHz}\right): \delta_{\mathrm{H}} 1.95-1.98\left(\mathrm{~m}, 4 \mathrm{H}, \mathrm{CH}_{2}\right), 2.06-2.11\left(\mathrm{~m}, 4 \mathrm{H}, \mathrm{CH}_{2}\right), 3.52\left(\mathrm{t}, J=6.3 \mathrm{~Hz}, 4 \mathrm{H}, \mathrm{CH}_{2}\right)$, 3.97-4.02 (m, 4H, $\left.\mathrm{CH}_{2}\right), 6.78-6.81(\mathrm{~m}, 4 \mathrm{H}, \mathrm{Ar}-\mathrm{H}), 6.98$ (d, J = 8.7 Hz, 2H, Ar-H), 7.16-7.30 (m, 8H, Ar-H), 7.40 (d, $J=8.7 \mathrm{~Hz}, 2 \mathrm{H}, \mathrm{Ar}-\mathrm{H}), 7.61$ (d, $J=7.3 \mathrm{~Hz}, 2 \mathrm{H}, \mathrm{Ar}-\mathrm{H}) .{ }^{13} \mathrm{C} \mathrm{NMR}\left(\mathrm{CDCl}_{3}, 100 \mathrm{MHz}\right): \delta_{\mathrm{C}} 26.79,28.40,28.43,32.33$, $32.42,65.76,66.00\left(\mathrm{CH}_{2}\right), 113.05,113.65,125.50,126.39,126.82,127.11,127.31,128.48,130.15,133.55,133.58$, 136.91, 157.77, 157.97. Anal.Calc. for $\mathrm{C}_{35} \mathrm{H}_{34} \mathrm{Br}_{2} \mathrm{~N}_{2} \mathrm{O}_{2}$ : C, 62.33; H, 5.08; N, 4.15\%. Found: C, 62.45; H, 4.98; N, $4.31 \%$. 

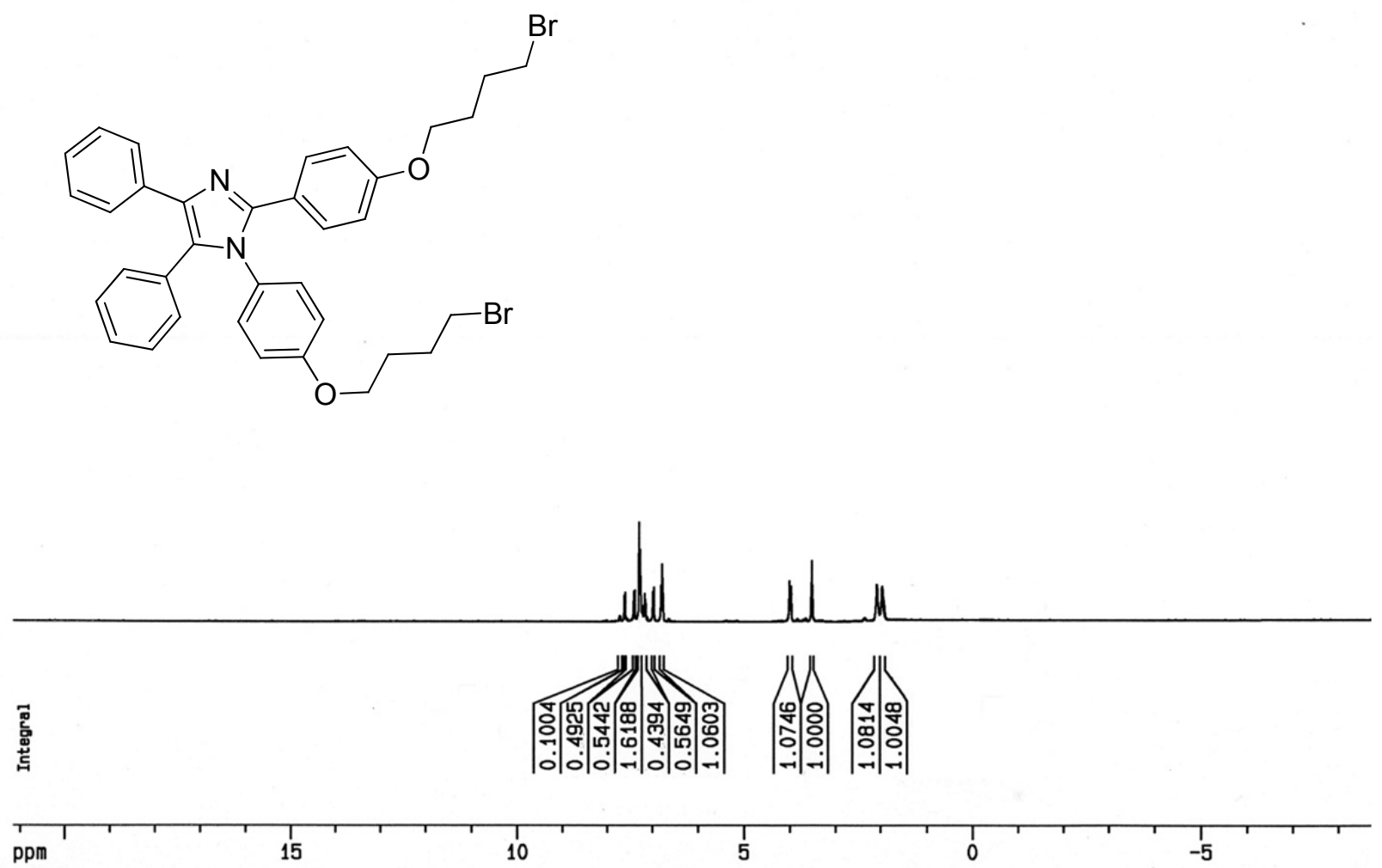

${ }^{1} \mathrm{H}$ NMR spectrum of 1,2-bis(4-(4-bromobutoxy)phenyl)-4,5-diphenyl-1 $\mathrm{H}$-imidazole (2d) in $\mathrm{CDCl}_{3}$ 

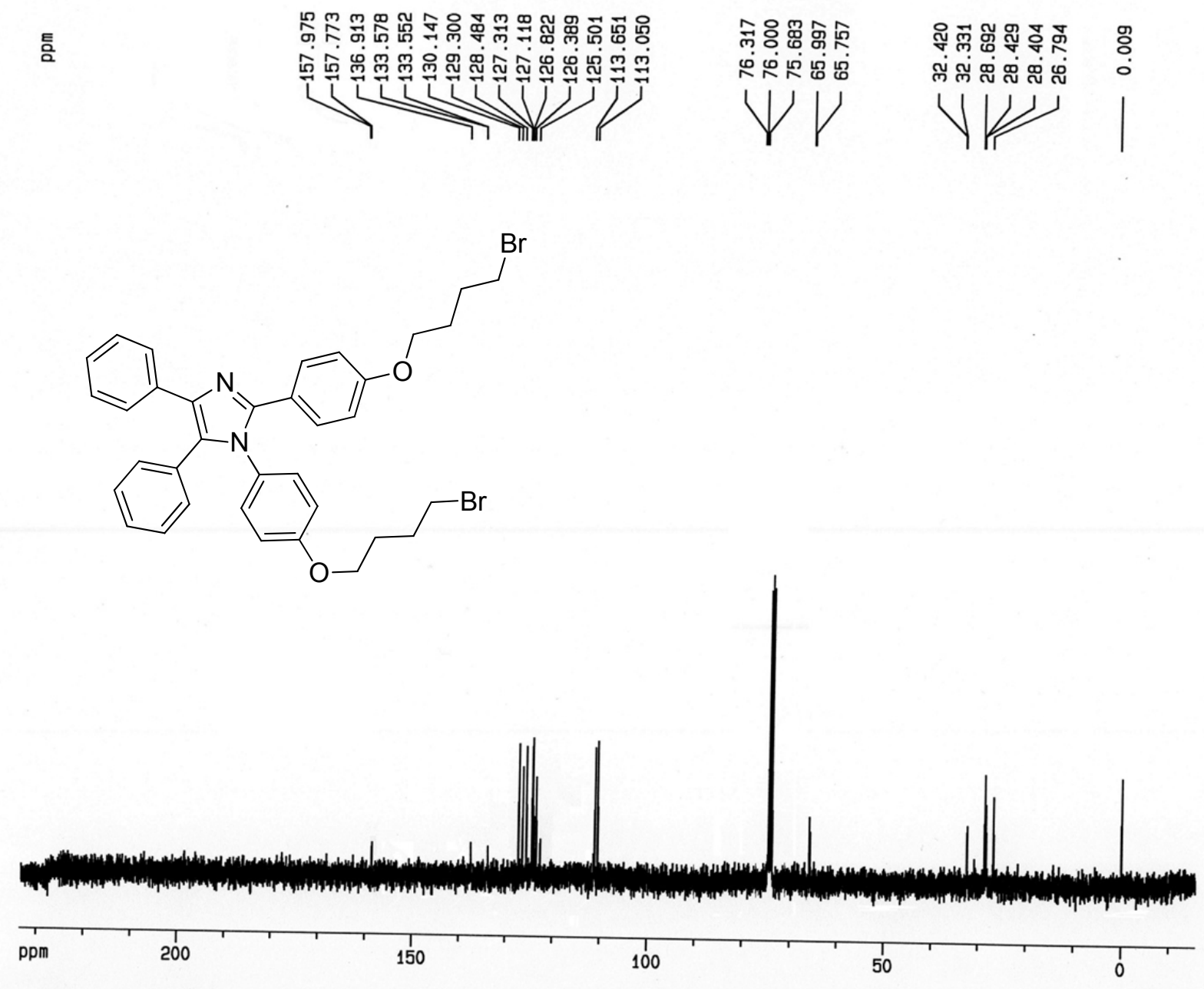

${ }^{13} \mathrm{C}$ NMR spectrum of 1,2-bis(4-(4-bromobutoxy)phenyl)-4,5-diphenyl- $1 \mathrm{H}$-imidazole (2d) in $\mathrm{CDCl}_{3}$ 


\section{2-(4-(3-bromopropoxy)phenyl)-4,5-diphenyl-1-p-tolyl-1H-imidazole (3b)}

White powder $(60 \%)$, M.P. $=160-162{ }^{\circ} \mathrm{C}, \mathrm{IR}\left(\mathrm{KBr}, \mathrm{cm}^{-1}\right)=3056,2924,1605,1577,1513,1465,1245,1175,1108$, 1026, 698. ${ }^{1} \mathrm{H} \mathrm{NMR}\left(\mathrm{CDCl}_{3}, 400 \mathrm{MHz}\right): \delta_{\mathrm{H}} 2.30-2.35\left(\mathrm{~m}, 5 \mathrm{H}, \mathrm{CH}_{2}\right.$ and $\left.\mathrm{CH}_{3}\right), 3.62\left(\mathrm{t}, J=6.4 \mathrm{~Hz}, 2 \mathrm{H}, \mathrm{CH}_{2}\right), 4.11(\mathrm{t}$, $\left.J=5.8 \mathrm{~Hz}, 2 \mathrm{H}, \mathrm{CH}_{2}\right), 6.81(\mathrm{~d}, J=8.6 \mathrm{~Hz}, 2 \mathrm{H}, \mathrm{Ar}-\mathrm{H}), 6.95$ (d, $\left.J=8.0 \mathrm{~Hz}, 2 \mathrm{H}, \mathrm{Ar}-\mathrm{H}\right), 7.09$ (d, J = 7.9 Hz, 2H, Ar-H), 7.15-7.30 (m, 8H, Ar-H), 7.40 (d, $J=8.6 \mathrm{~Hz}, 2 \mathrm{H}, \mathrm{Ar}-\mathrm{H}), 7.62(\mathrm{~d}, J=7.3 \mathrm{~Hz}, 2 \mathrm{H}, \mathrm{Ar}-\mathrm{H}) .{ }^{13} \mathrm{C}$ NMR $\left(\mathrm{CDCl}_{3}, 100\right.$ MHz): $\delta_{\mathrm{C}} 20.15\left(\mathrm{CH}_{3}\right), 28.93,31.30,64.20\left(\mathrm{CH}_{2}\right), 113.05,122.46,125.46,126.37,126.53,126.78,127.09,127.13$, $127.26,128.19,128.67,129.30,129.55,129.82,130.12,133.54,136.90,137.07,145.82,157.61$. Anal.Calcd for $\mathrm{C}_{31} \mathrm{H}_{27} \mathrm{BrN}_{2} \mathrm{O}: \mathrm{C}, 71.13 ; \mathrm{H}, 5.20 ; \mathrm{N}, 5.35 \%$. Found: $\mathrm{C}, 70.98 ; \mathrm{H}, 5.04 ; \mathrm{N}, 5.24 \%$. 
틈

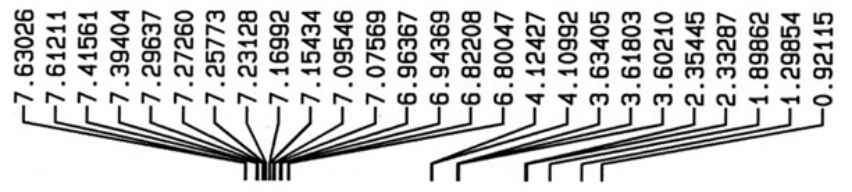<smiles>Cc1ccc(-n2c(-c3ccc(OCCCBr)cc3)nc(-c3ccccc3)c2-c2ccccc2)cc1</smiles>
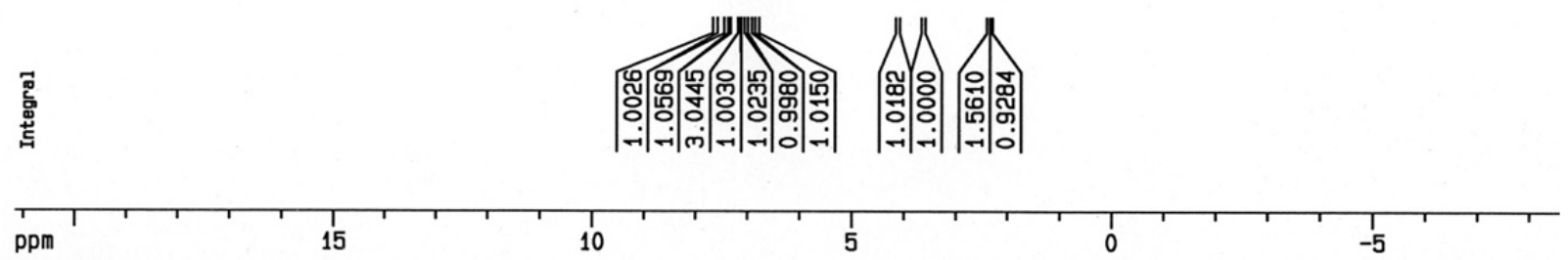

${ }^{1} \mathrm{H}$ NMR spectrum of 2-(4-(3-bromopropoxy)phenyl)-4,5-diphenyl-1-p-tolyl-1H-imidazole (3b) in $\mathrm{CDCl}_{3}$ 

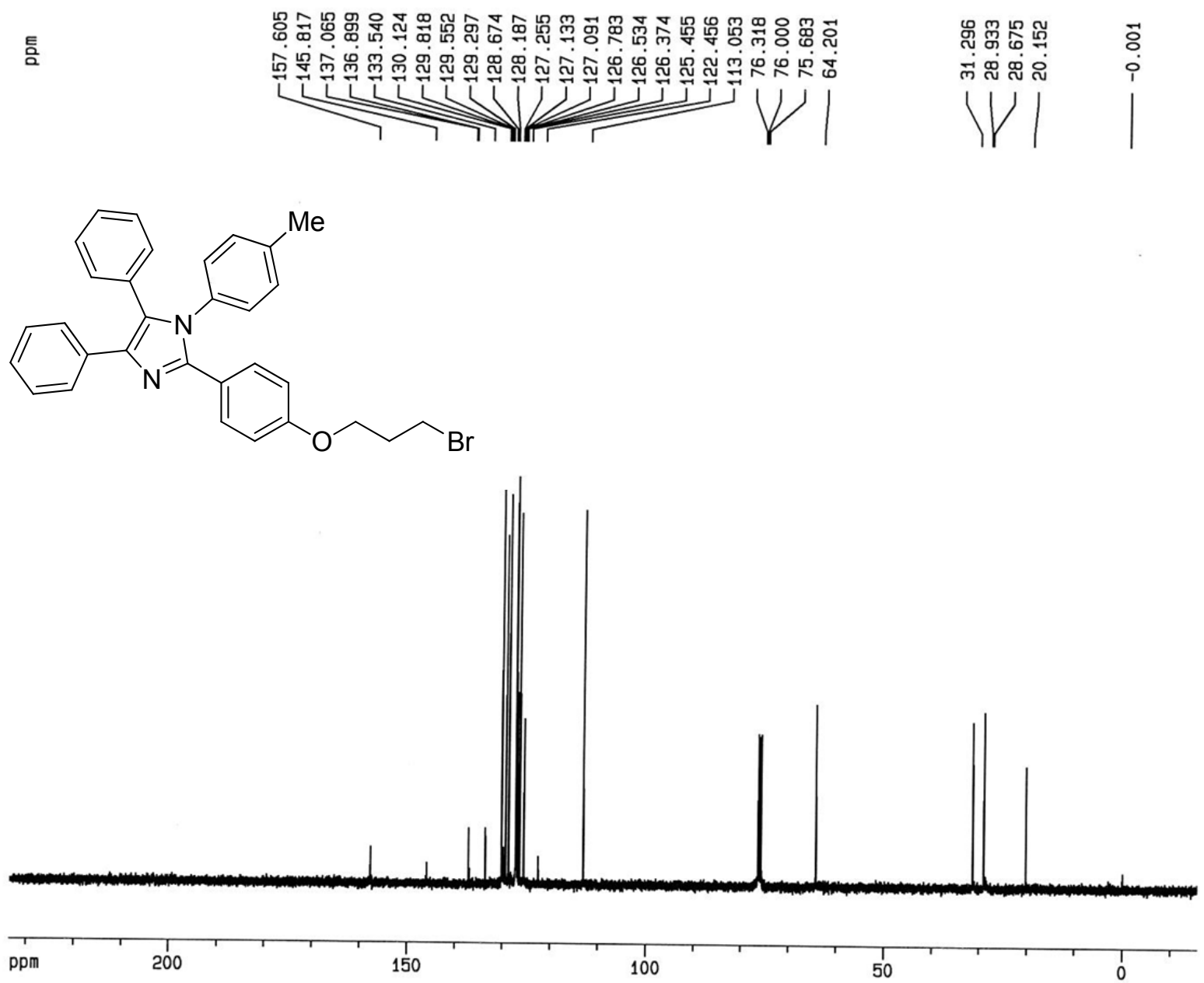

${ }^{13} \mathrm{C}$ NMR spectrum of 2-(4-(3-bromopropoxy)phenyl)-4,5-diphenyl-1-p-tolyl- $1 \mathrm{H}$-imidazole (3b) in $\mathrm{CDCl}_{3}$ 


\section{2-(4-(4-bromobutoxy)phenyl)-4,5-diphenyl-1-p-tolyl-1H-imidazole (4b)}

White powder $(62 \%)$, M.P. $=182-184{ }^{\circ} \mathrm{C}, \mathrm{IR}\left(\mathrm{KBr}, \mathrm{cm}^{-1}\right)=3058,2927,1639,1616,1513,1469,1241,1015,829$, 776, 699. ${ }^{1} \mathrm{H}$ NMR $\left(\mathrm{CDCl}_{3}, 400 \mathrm{MHz}\right): \delta_{\mathrm{H}} 1.90-1.95$ (m, 2H, $\left.\mathrm{CH}_{2}\right), 2.01-2.08\left(\mathrm{~m}, 2 \mathrm{H}, \mathrm{CH}_{2}\right), 2.31\left(\mathrm{~s}, 3 \mathrm{H}, \mathrm{CH}_{3}\right), 3.48$ (t, $\left.J=6.6 \mathrm{~Hz}, 2 \mathrm{H}, \mathrm{CH}_{2}\right), 3.96\left(\mathrm{t}, J=6.0 \mathrm{~Hz}, 2 \mathrm{H}, \mathrm{CH}_{2}\right), 6.75(\mathrm{~d}, J=8.8 \mathrm{~Hz}, 2 \mathrm{H}, \mathrm{Ar}-\mathrm{H}), 6.91(\mathrm{~d}, J=8.1 \mathrm{~Hz}, 2 \mathrm{H}, \mathrm{Ar}-$ H), 7.04 (d, $J=8.1 \mathrm{~Hz}, 2 \mathrm{H}, \mathrm{Ar}-\mathrm{H}), 7.11-7.26(\mathrm{~m}, 8 \mathrm{H}, \mathrm{Ar}-\mathrm{H}), 7.35$ (d, J= 8.8 Hz, 2H, ArH), 7.58 (d, J= 7.3 Hz, 2H, Ar-H). ${ }^{13} \mathrm{C}$ NMR $\left(\mathrm{CDCl}_{3}, 100 \mathrm{MHz}\right): \delta_{\mathrm{C}} 20.17\left(\mathrm{CH}_{3}\right), 26.79,28.40,32.47,65.69\left(\mathrm{CH}_{2}\right), 112.95,125.44,126.37$, $126.76,127.11,127.26,128.66,129.28,129.49,129.80,130.11,133.53,137.03,145.86,157.75$. Anal.Calc. for $\mathrm{C}_{32} \mathrm{H}_{29} \mathrm{BrN}_{2} \mathrm{O}: \mathrm{C}, 71.51 ; \mathrm{H}, 5.44 ; \mathrm{N}, 5.21 \%$. Found: $\mathrm{C}, 71.39 ; \mathrm{H}, 5.51 ; \mathrm{N}, 5.24 \%$. 
틈

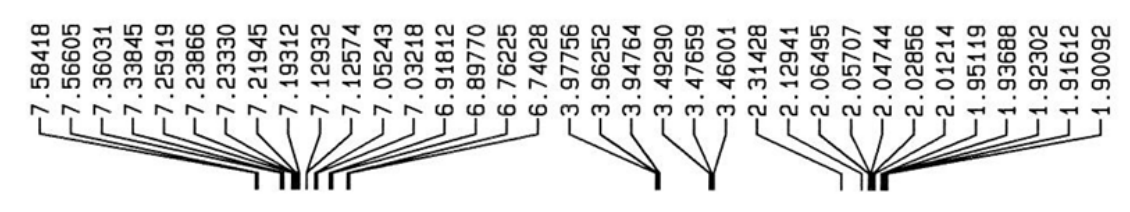<smiles>Cc1ccc(-n2c(-c3ccccc3)nc(-c3ccccc3)c2-c2ccc(OCCCCBr)cc2)cc1</smiles>

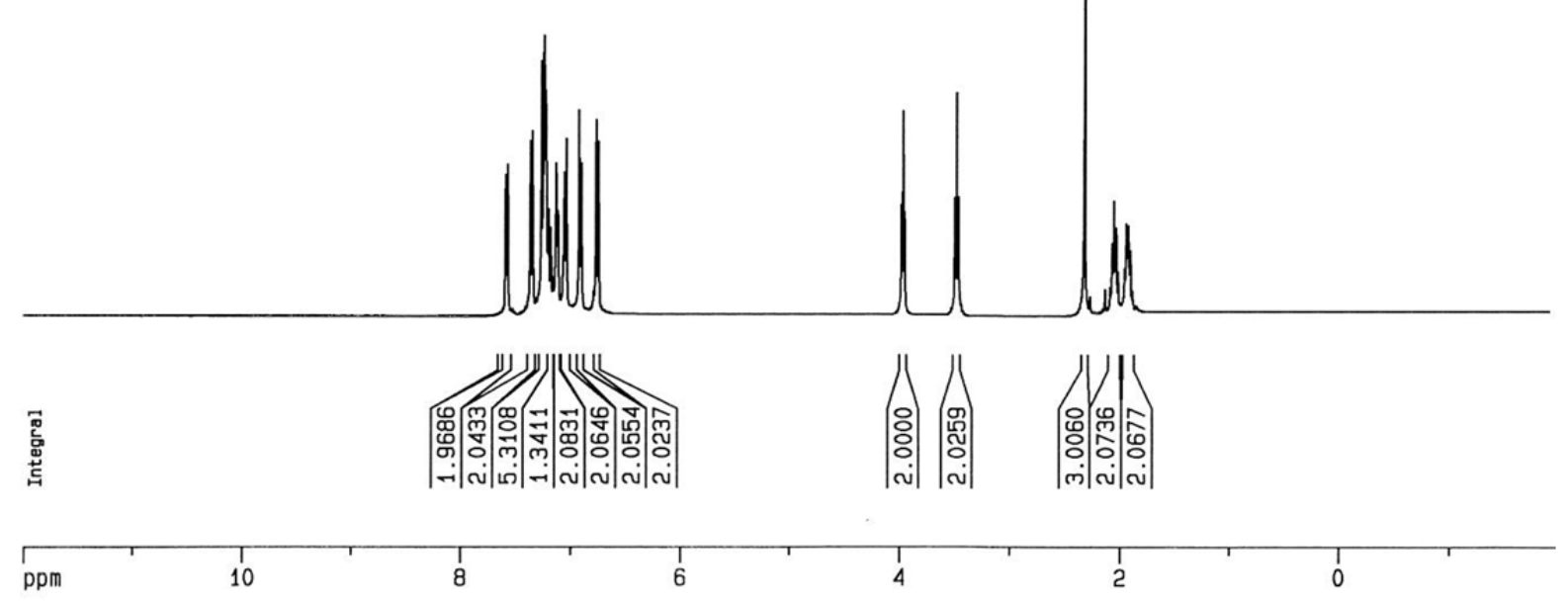

${ }^{1} \mathrm{H}$ NMR spectrum of 2-(4-(4-bromobutoxy)phenyl)-4,5-diphenyl-1-p-tolyl-1H-imidazole (4b) in $\mathrm{CDCl}_{3}$ 

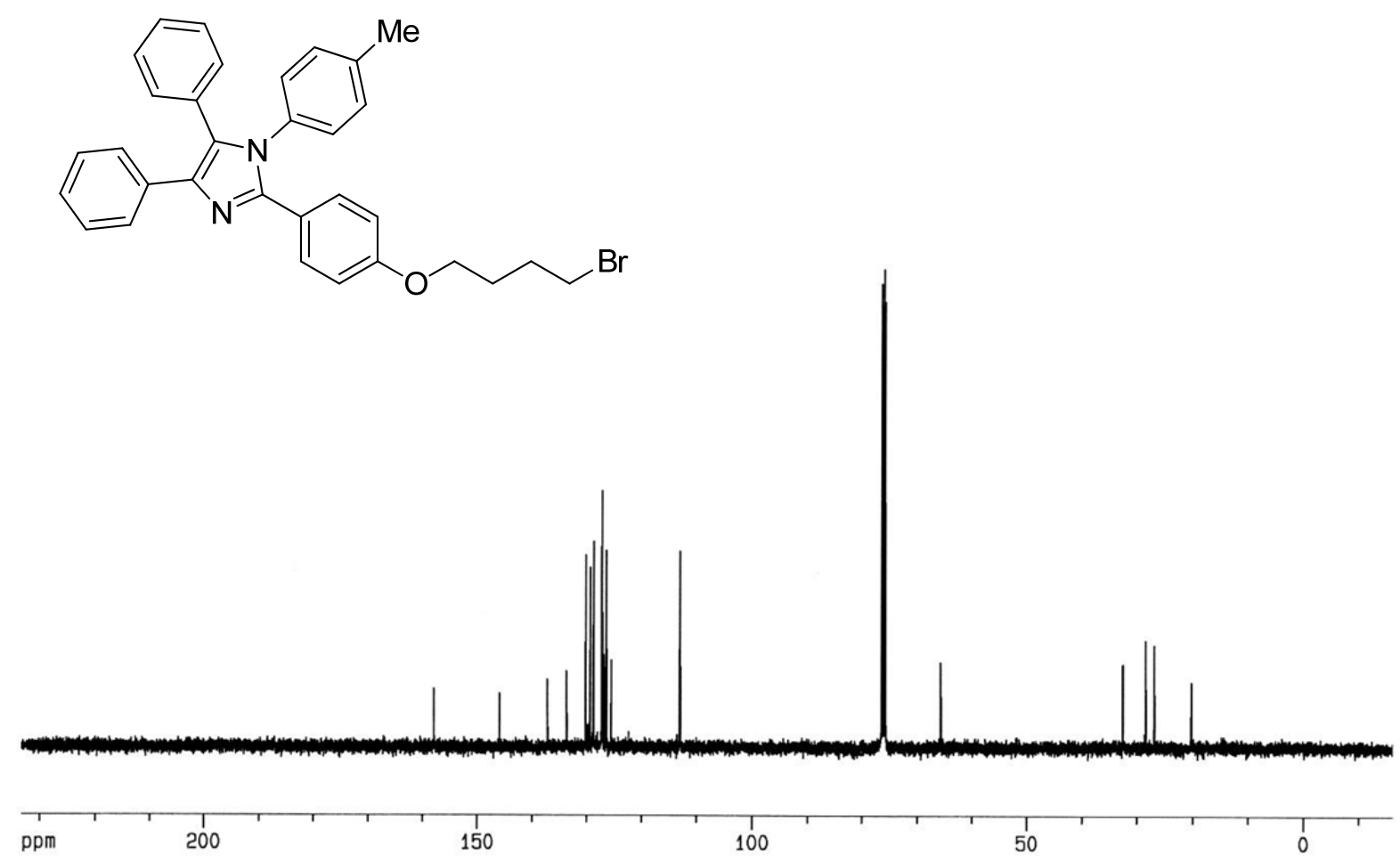

${ }^{13} \mathrm{C}$ NMR spectrum of 2-(4-(4-bromobutoxy)phenyl)-4,5-diphenyl-1-p-tolyl-1 $H$-imidazole (4b) in $\mathrm{CDCl}_{3}$ 


\section{General procedure for preparation of imidazoles containing bulky organosilicon groups}

To a stirred solution of $\left(\mathrm{Me}_{3} \mathrm{Si}\right)_{3} \mathrm{CLi}(5 \mathrm{mmol})$ in THF was added a bromo-imidazole derivative $(5 \mathrm{mmol})$ in $10 \mathrm{ml}$ THF and or $(2.5 \mathrm{mmol})$ of $\mathbf{2 d}$ compound in $\mathrm{THF}$ at ${ }^{\circ} \mathrm{C}$. The mixture was stirred for another 10 min at room temperature. The mixture was poured into ammonium chloride solution $(50 \mathrm{ml})$ and extracted with $\mathrm{CH}_{2} \mathrm{Cl}_{2}(2 \times 20$ $\mathrm{ml})$. The organic phase was washed with water $(100 \mathrm{~mL})$ and dried $\left(\mathrm{Na}_{2} \mathrm{SO}_{4}\right)$, and the solvent was removed in vacuum to yield a yellow solid.

Selected spectral data of the products:

\section{1-(4-(5,5,5-tris(trimethylsilyl)pentyloxy)phenyl)-2,4,5-triphenyl-1H-imidazole (3a)}

White powder $(90 \%)$, M.P. $=164-166^{\circ} \mathrm{C}, \mathrm{IR}\left(\mathrm{KBr}, \mathrm{cm}^{-1}\right)=3061,2953,1611,1510,1471,1445,1255,1029,840$, 695, 673, 641, 615, 536. ${ }^{1} \mathrm{H}$ NMR $\left(\mathrm{CDCl}_{3}, 400 \mathrm{MHz}\right): \delta_{\mathrm{H}} 0.15\left(\mathrm{~s}, 27 \mathrm{H}, \mathrm{SiMe}_{3}\right), 1.70-1.79\left(\mathrm{~m}, 6 \mathrm{H}, 3 \times \mathrm{CH}_{2}\right), 3.93(\mathrm{t}, J$ $\left.=6.0 \mathrm{~Hz}, 2 \mathrm{H}, \mathrm{CH}_{2}\right), 6.77(\mathrm{~d}, J=8.8 \mathrm{~Hz}, 2 \mathrm{H}, \mathrm{Ar}-\mathrm{H}), 6.98(\mathrm{~d}, J=8.8 \mathrm{~Hz}, 2 \mathrm{H}, \mathrm{Ar}-\mathrm{H}), 7.16-7.36(\mathrm{~m}, 11 \mathrm{H}, \mathrm{Ar}-\mathrm{H}), 7.47-$ $7.50(\mathrm{~m}, 2 \mathrm{H}, \mathrm{Ar}-\mathrm{H}), 7.62(\mathrm{~d}, J=7.1 \mathrm{~Hz}, 2 \mathrm{H}, \mathrm{Ar}-\mathrm{H}) .{ }^{13} \mathrm{C} \mathrm{NMR}\left(\mathrm{CDCl}_{3}, 100 \mathrm{MHz}\right): \delta_{\mathrm{C}} 1.75\left(\mathrm{SiMe}_{3}\right), 5.14$ $\left(\mathrm{C}\left(\mathrm{SiMe}_{3}\right)_{3}\right), 25.46,29.52,29.83,66.60\left(\mathrm{CH}_{2}\right), 113.67,125.50,126.38,126.84,127.07,127.11,127.31,127.90$ 128.10, 128.38, 128.70, 129.66, 129.79, 130.14, 133.55, 137.09, 157.57. Anal.Calc. for $\mathrm{C}_{41} \mathrm{H}_{54} \mathrm{~N}_{2} \mathrm{OSi}_{3}$ : C, $72.94 ; \mathrm{H}_{\text {, }}$ 8.06; N, 4.15. Found: C, 72.79; H, 8.11; N, 3.94\%. 


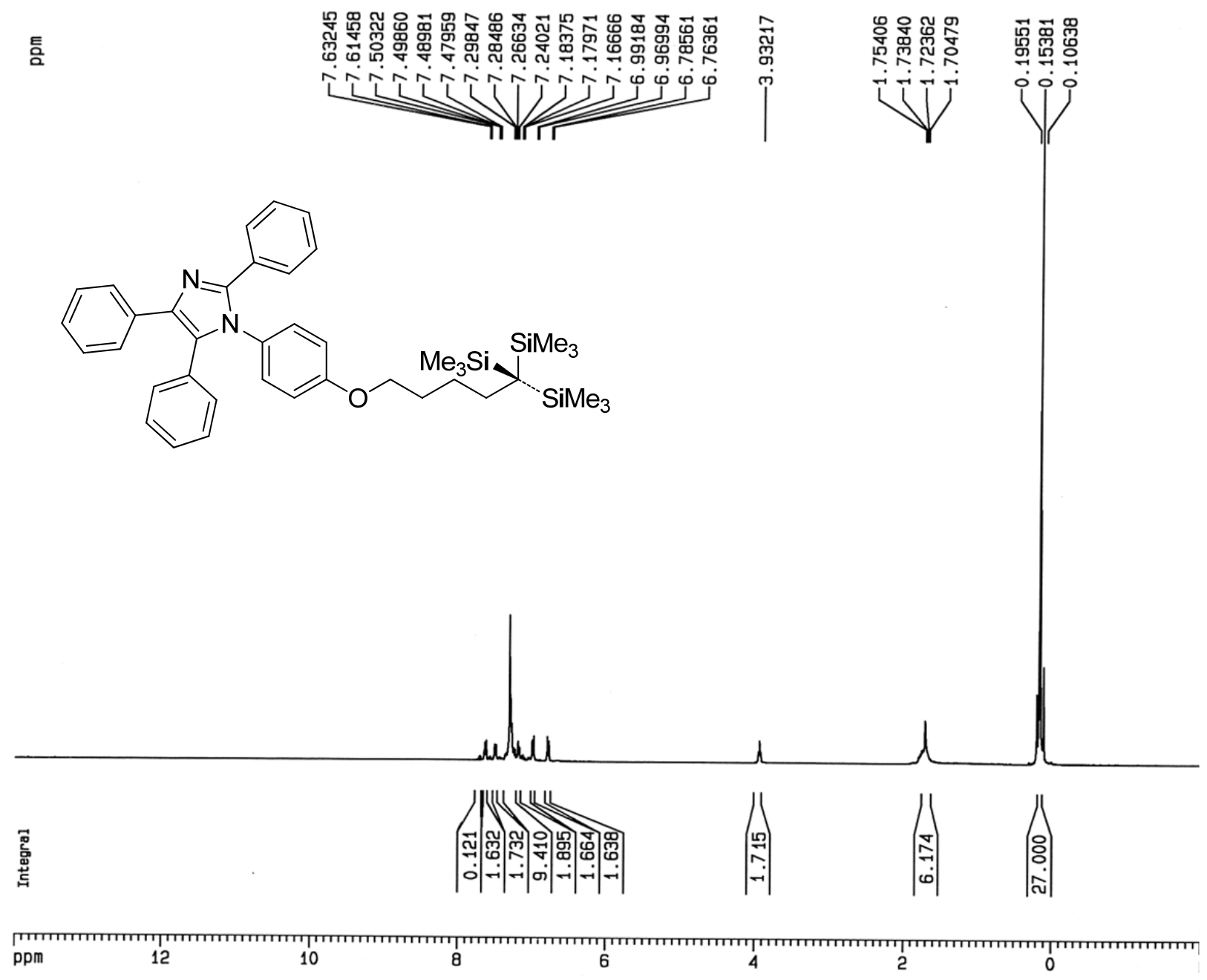

${ }^{1} \mathrm{H}$ NMR spectrum of 1-(4-(5,5,5-tris(trimethylsilyl)pentyloxy)phenyl)-2,4,5-triphenyl-1H-imidazole (3a) in $\mathrm{CDCl}_{3}$ 
<smiles>C[SiH3]C([SiH3])([SiH3])CCCOc1ccc(-n2c(-c3ccccc3)nc(-c3ccccc3)c2-c2ccccc2)cc1</smiles>

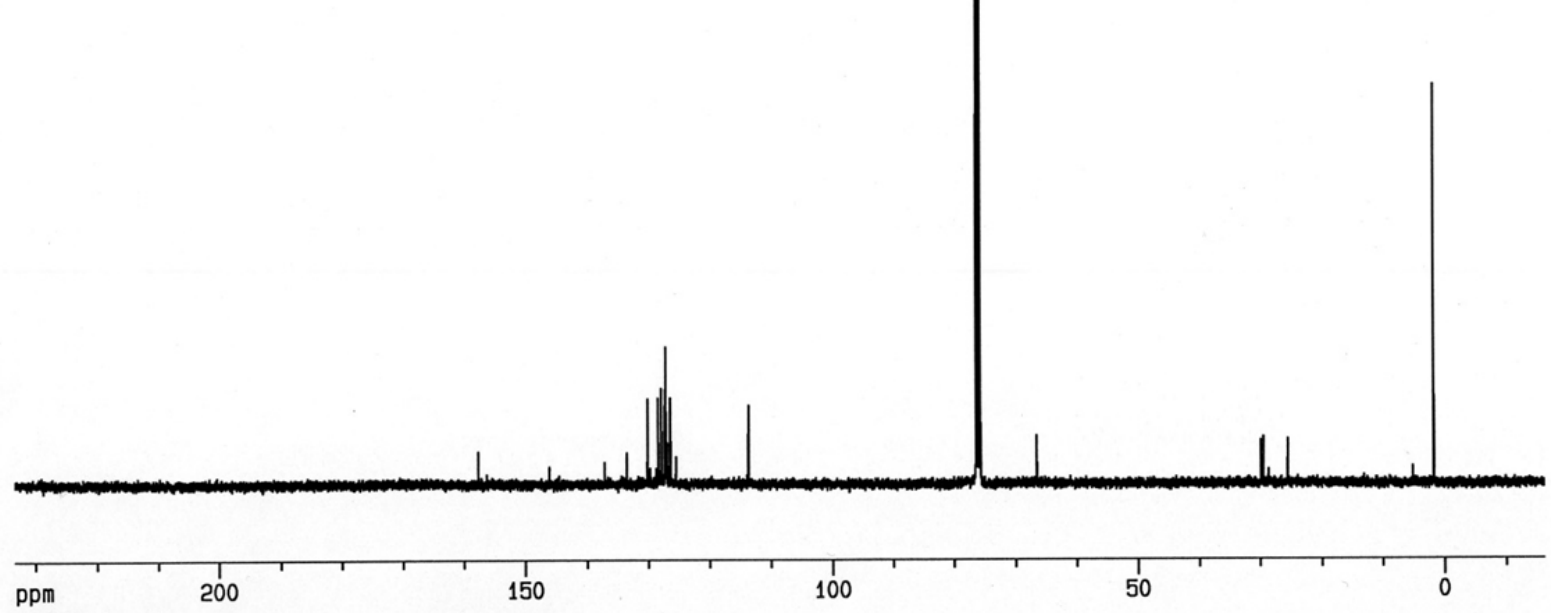

${ }^{13} \mathrm{C}$ NMR spectrum of 1-(4-(5,5,5-tris(trimethylsilyl)pentyloxy)phenyl)-2,4,5-triphenyl-1H-imidazole (3a) in $\mathrm{CDCl}_{3}$ 


\section{1-(4-(5,5,5-tris(trimethylsilyl)pentyloxy)phenyl)-2-(4-chlorophenyl)-4,5-diphenyl-1H-imidazole (3c)}

White powder (91\%), M.P. $=168-170{ }^{\circ} \mathrm{C}, \mathrm{IR}\left(\mathrm{KBr}, \mathrm{cm}^{-1}\right)=3059,2958,1618,1541,1512,1473,1256,1094,840$, 620. ${ }^{1} \mathrm{H}$ NMR $\left(\mathrm{CDCl}_{3}, 400 \mathrm{MHz}\right): \delta_{\mathrm{H}} 0.10\left(\mathrm{~s}, 27 \mathrm{H}, \mathrm{SiMe}_{3}\right), 1.62-1.66\left(\mathrm{~m}, 6 \mathrm{H}, 3 \times \mathrm{CH}_{2}\right), 3.85\left(\mathrm{t}, J=5.9 \mathrm{~Hz}, 2 \mathrm{H}, \mathrm{CH}_{2}\right)$, $6.69(\mathrm{~d}, J=8.8 \mathrm{~Hz}, 2 \mathrm{H}, \mathrm{Ar}-\mathrm{H}), 6.88$ (d, $J=8.8 \mathrm{~Hz}, 2 \mathrm{H}, \mathrm{Ar}-\mathrm{H}), 7.06$ (dd, $J=1.7 \mathrm{~Hz}, J=7.42 \mathrm{~Hz}, 2 \mathrm{H}, \mathrm{Ar}-\mathrm{H}), 7.11-$ $7.23(\mathrm{~m}, 8 \mathrm{H}, \mathrm{Ar}-\mathrm{H}), 7.33$ (d, J= 8.5 Hz, 2H, Ar-H), 7.50 (d, J= $7.2 \mathrm{~Hz}, 2 \mathrm{H}, \mathrm{Ar}-\mathrm{H}) .{ }^{13} \mathrm{C}$ NMR $\left(\mathrm{CDCl}_{3}, 100 \mathrm{MHz}\right): \delta_{\mathrm{C}}$ $1.72\left(\mathrm{SiMe}_{3}\right), 4.43\left(\mathrm{C}\left(\mathrm{SiMe}_{3}\right)_{3}\right), 25.46,29.51,29.83,66.60\left(\mathrm{CH}_{2}\right), 113.95,126.18,126.37,126.59,127.28,127.50$, 127.53, 128.02, 128.24, 128.42, 128.81, 128.87, 129.38, 129.68, 130.09, 130.12, 133.00, 137.04, 157.31. Anal.Calc. for $\mathrm{C}_{41} \mathrm{H}_{53} \mathrm{ClN}_{2} \mathrm{OSi}_{3}: \mathrm{C}, 69.40 ; \mathrm{H}, 7.53 ; \mathrm{N}, 3.95 \%$. Found: $\mathrm{C}, 69.48 ; \mathrm{H}, 7.32 ; \mathrm{N}, 3.79 \%$. 


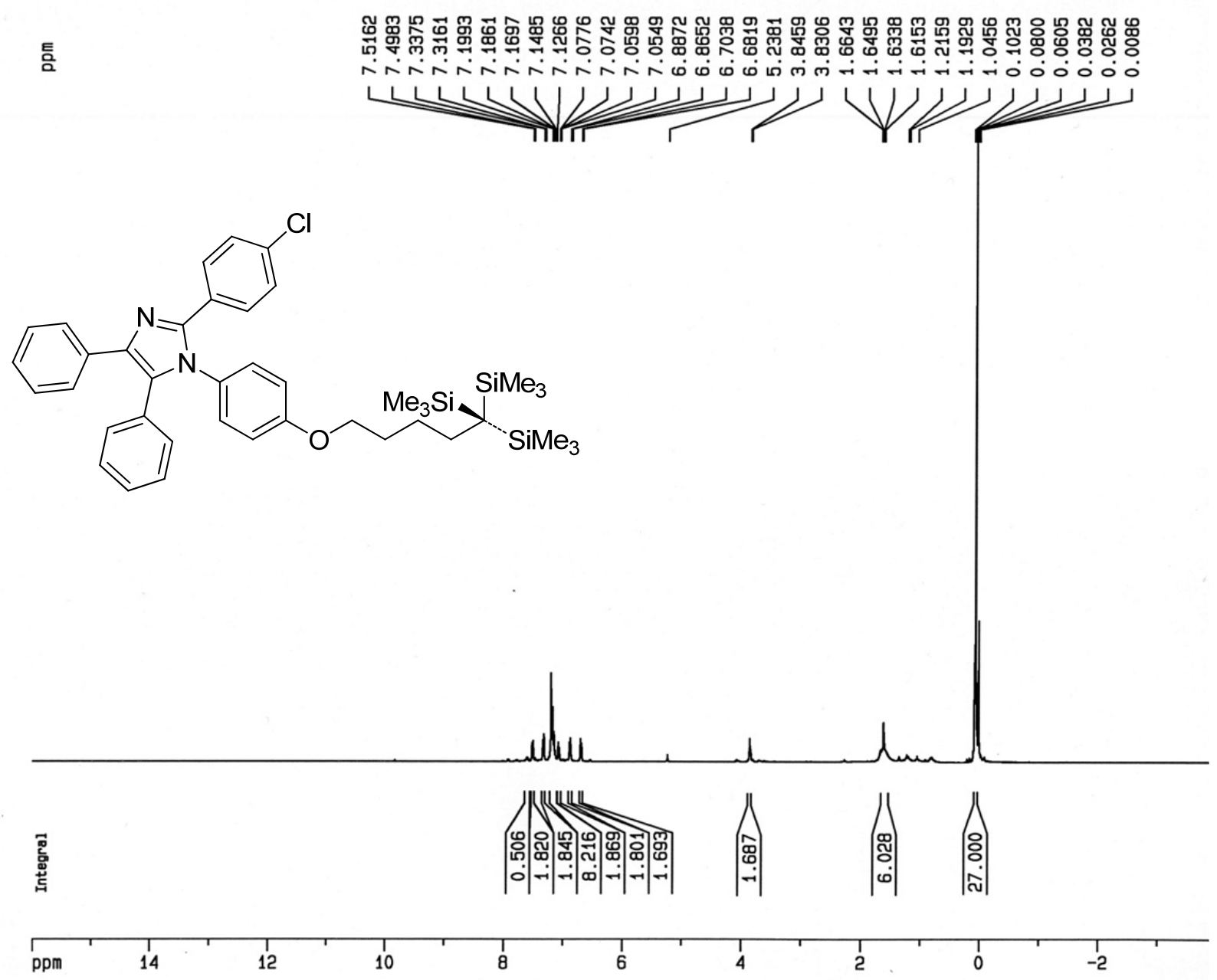

${ }^{1} \mathrm{H}$ NMR spectrum of 1-(4-(5,5,5-tris(trimethylsilyl)pentyloxy)phenyl)-2-(4-chlorophenyl)-4,5-diphenyl-1Himidazole (3c) in $\mathrm{CDCl}_{3}$ 
<smiles>CCCC([SiH3])([SiH3])[SiH2]CCCOc1ccc(-n2c(-c3ccc(Cl)cc3)nc(-c3ccccc3)c2-c2ccccc2)cc1</smiles>

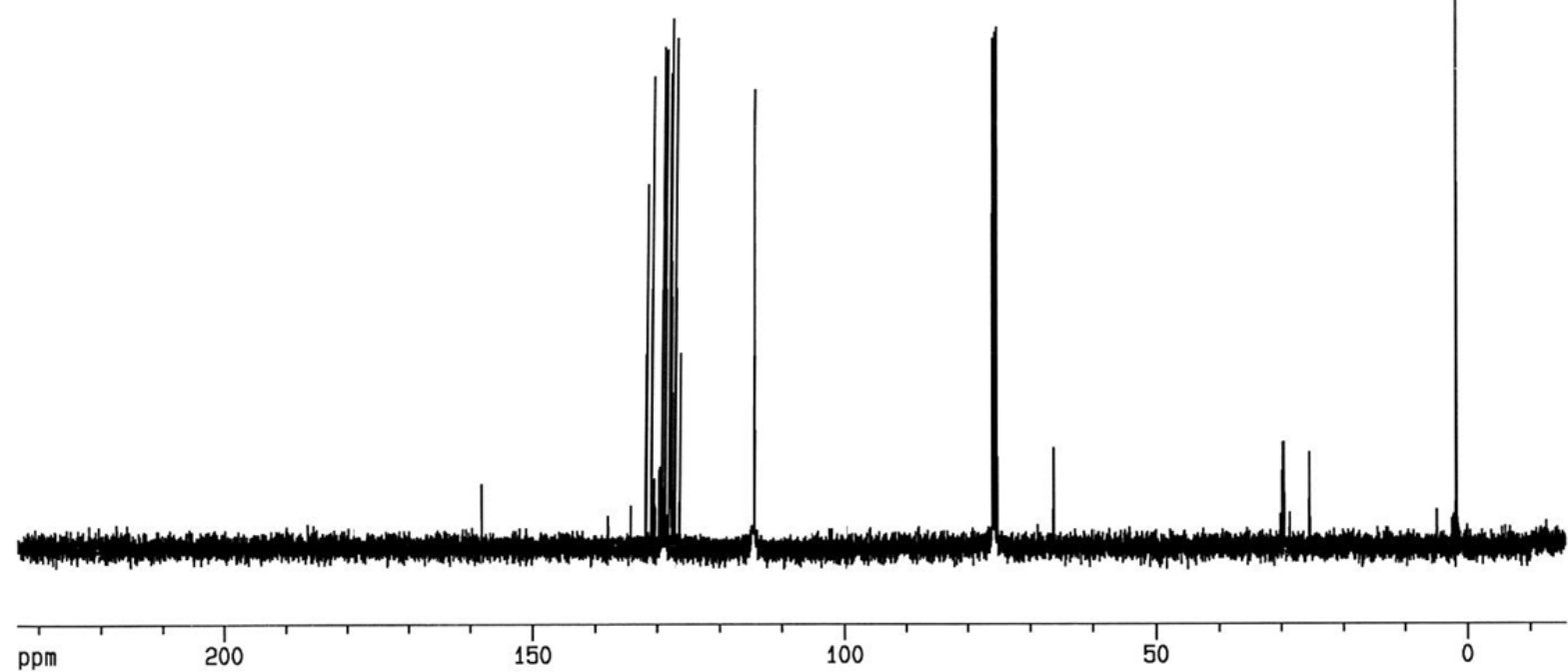

${ }^{13} \mathrm{C}$ NMR spectrum of 1-(4-(5,5,5-tris(trimethylsilyl)pentyloxy)phenyl)-2-(4-chlorophenyl)-4,5-diphenyl-1Himidazole (3c) in $\mathrm{CDCl}_{3}$ 


\section{1,2-bis(4-(5,5,5-tris(trimethylsilyl)pentyloxy)phenyl)-4,5-diphenyl-1H-imidazole (3d)}

White powder (89\%), M.P. $=200-202^{\circ} \mathrm{C}, \mathrm{IR}\left(\mathrm{KBr}, \mathrm{cm}^{-1}\right)=3058,2925,1617,1558,1540,1510,1457,1250,841$, 619. ${ }^{1} \mathrm{H}$ NMR $\left(\mathrm{CDCl}_{3}, 400 \mathrm{MHz}\right): \delta_{\mathrm{H}} 0.05$ (s, 27H, SiMe3), 0.06 (s, 27H, $\left.\mathrm{SiMe}_{3}\right), 1.54-1.63\left(\mathrm{~m}, 12 \mathrm{H}, \mathrm{CH}_{2}\right), 3.82-$ $3.87\left(\mathrm{~m}, 4 \mathrm{H}, \mathrm{CH}_{2}\right), 6.66-6.72(\mathrm{~m}, 4 \mathrm{H}, \mathrm{Ar}-\mathrm{H}), 6.88(\mathrm{~d}, J=8.8 \mathrm{~Hz}, 2 \mathrm{H}, \mathrm{Ar}-\mathrm{H}), 7.01-7.21(\mathrm{~m}, 8 \mathrm{H}, \mathrm{Ar}-\mathrm{H}), 7.30(\mathrm{~d}, J=$ 8.8, 2H, Ar-H), 7.52 (d, J = 7.1, 2H, Ar-H). ${ }^{13} \mathrm{C}$ NMR $\left(\mathrm{CDCl}_{3}, 100 \mathrm{MHz}\right): \delta_{\mathrm{C}} 1.75\left(\mathrm{SiMe}_{3}\right), 4.43\left(\mathrm{C}\left(\mathrm{SiMe}_{3}\right)_{3}\right), 25.51$, 28.69, 66.84, $65.99\left(\mathrm{CH}_{2}\right), 113.05,113.63,125.49,126.38,126.82,127.09,127.27,128.42,129.21,130.14,133.52$, 133.56, 136.83, 157.77, 157.97. Anal.Calc. for $\mathrm{C}_{55} \mathrm{H}_{88} \mathrm{~N}_{2} \mathrm{O}_{2} \mathrm{Si}_{6}$ : C, 67.56; H, 9.07; N, 2.86\%. Found: C, 67.44; $\mathrm{H}$, $9.28 ; \mathrm{N}, 2.65 \%$. 
<smiles>C[SiH3]C([SiH3])([SiH3])[SiH2]CCCCOc1ccc(-c2nc(-c3ccccc3)c(-c3ccccc3)n2-c2ccc(OCCCC[Si](C)(C)C(C)C)cc2)cc1</smiles>
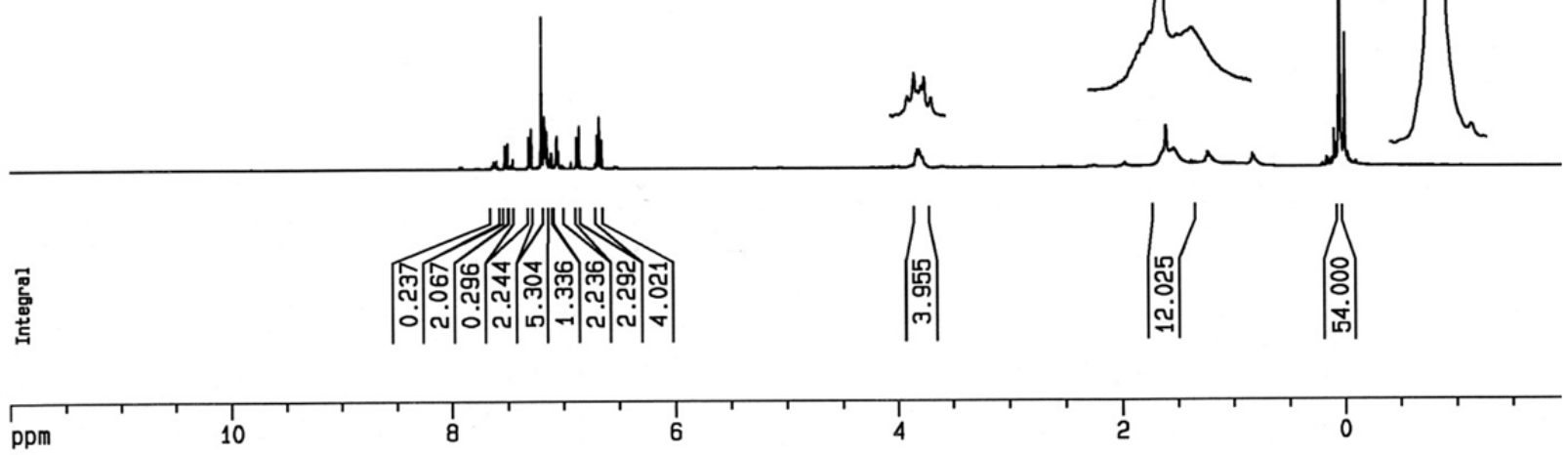

${ }^{1} \mathrm{H}$ NMR spectrum of 1,2-bis(4-(5,5,5-tris(trimethylsilyl)pentyloxy)phenyl)-4,5-diphenyl-1H-imidazole (3d) in $\mathrm{CDCl}_{3}$ 

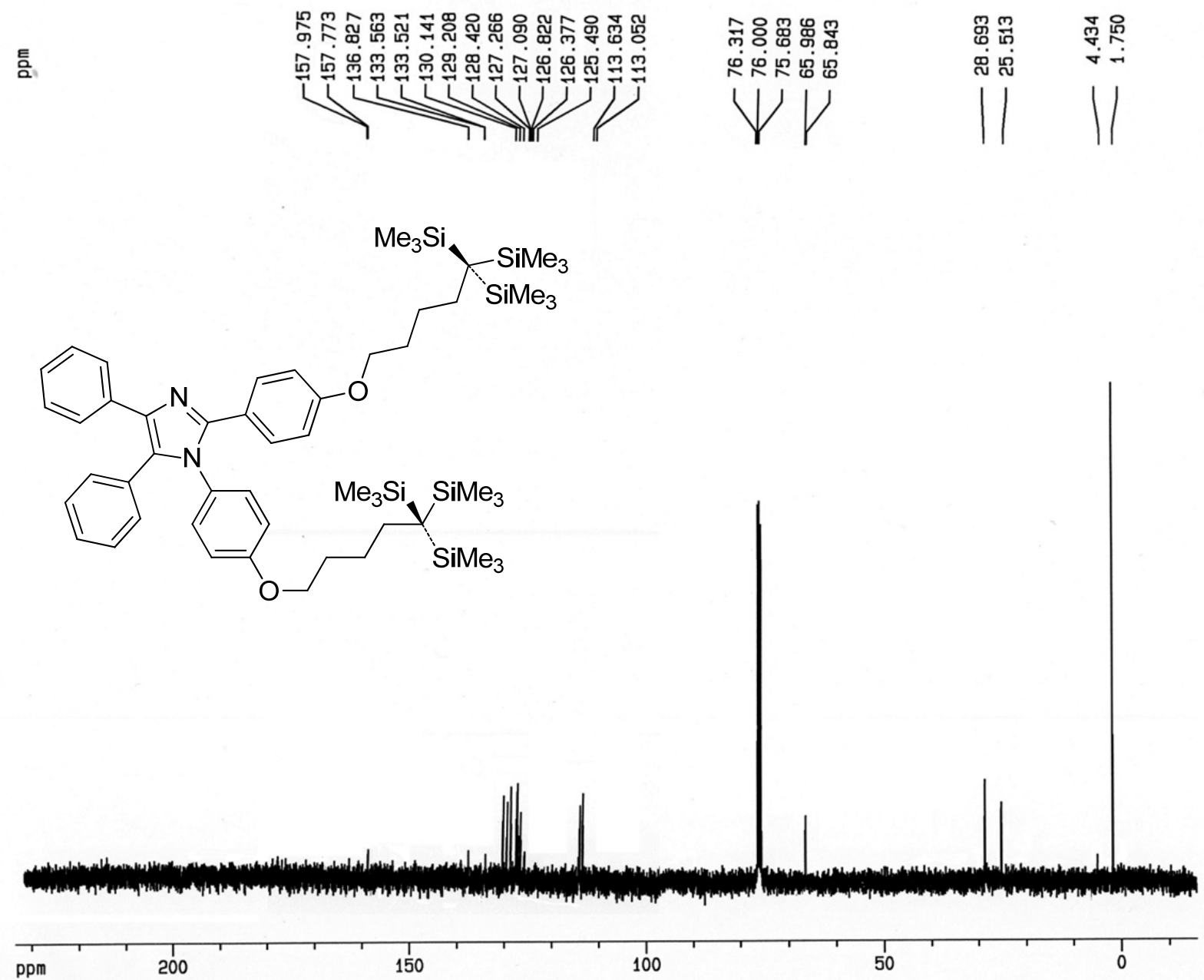

${ }^{13} \mathrm{C}$ NMR spectrum of 1,2-bis(4-(5,5,5-tris(trimethylsilyl)pentyloxy)phenyl)-4,5-diphenyl- $1 \mathrm{H}$-imidazole (3d) in $\mathrm{CDCl}_{3}$ 


\section{2-(4-(3,3,3-tris(trimethylsilyl)propoxy)phenyl)-4,5-diphenyl-1-p-tolyl-1H-imidazole (5b)}

White powder $(89 \%)$, M.P. $=106-108^{\circ} \mathrm{C}, \mathrm{IR}\left(\mathrm{KBr}, \mathrm{cm}^{-1}\right)=3058,2955,1609,1578,1512,1475,1422,1256,1024$, 845, 696. ${ }^{1} \mathrm{H}$ NMR $\left(\mathrm{CDCl}_{3}, 400 \mathrm{MHz}\right): \delta_{\mathrm{H}} 0.18$ (s, 27H, $\left.\mathrm{SiMe}_{3}\right), 2.15$ (t, J= 8.2 Hz, 2H, $\left.\mathrm{CH}_{2}\right), 2.34$ (s, 3H, $\mathrm{CH}_{3}$ ), $4.08\left(\mathrm{t}, J=8.2 \mathrm{~Hz}, 2 \mathrm{H}, \mathrm{CH}_{2}\right), 6.74(\mathrm{~d}, J=8.8 \mathrm{~Hz}, 2 \mathrm{H}, \mathrm{Ar}-\mathrm{H}), 6.93(\mathrm{~d}, J=8.2 \mathrm{~Hz}, 2 \mathrm{H}, \mathrm{Ar}-\mathrm{H}), 7.07$ (d, $J=8.2 \mathrm{~Hz}$, 2H, Ar-H), 7.13-7.28 (m, 8H, Ar-H), 7.37 (d, $J=7.9 \mathrm{~Hz}, 2 \mathrm{H}, \mathrm{Ar}-\mathrm{H}), 7.60$ (d, $J=7.9 \mathrm{~Hz}, 2 \mathrm{H}, \mathrm{Ar}-\mathrm{H}) .{ }^{13} \mathrm{C}$ NMR $\left(\mathrm{CDCl}_{3}, 100 \mathrm{MHz}\right): \delta_{\mathrm{C}} 1.72\left(\mathrm{SiMe}_{3}\right), 4.40\left(\mathrm{C}\left(\mathrm{SiMe}_{3}\right)_{3}\right), 20.18\left(\mathrm{CH}_{3}\right), 29.10,67.49\left(\mathrm{CH}_{2}\right), 113.06,125.42,126.36$, $126.76,127.09,127.12,127.26,128.67,129.33,129.89,130.14,133.63,137.02,146.00,158.00$. Anal.Calc. for $\mathrm{C}_{40} \mathrm{H}_{52} \mathrm{~N}_{2} \mathrm{OSi}_{3}:$ C, 72.67; H, 7.93; N, 4.24\%. Found: C, 72.46; H, 7.85; N, 4.11\%. 


\section{틈}
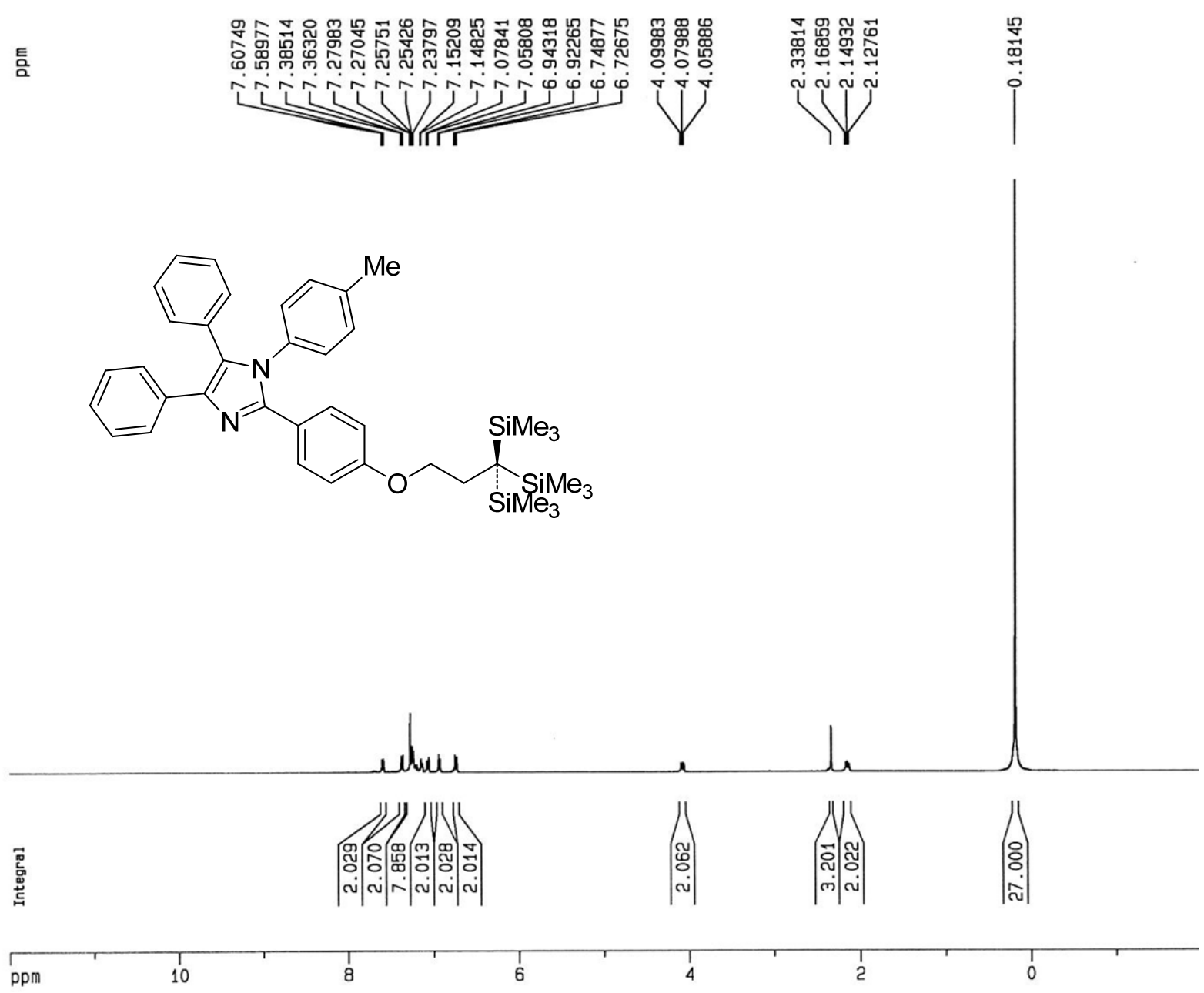

${ }^{1} \mathrm{H}$ NMR spectrum of 2-(4-(3,3,3-tris(trimethylsilyl)propoxy)phenyl)-4,5-diphenyl-1-p-tolyl-1H-imidazole (5b) in $\mathrm{CDCl}_{3}$ 
<smiles>C[SiH2][C@@H](C)CCOc1ccc(-c2nc(-c3ccccc3)c(-c3ccccc3)n2-c2ccc(C)cc2)cc1</smiles>

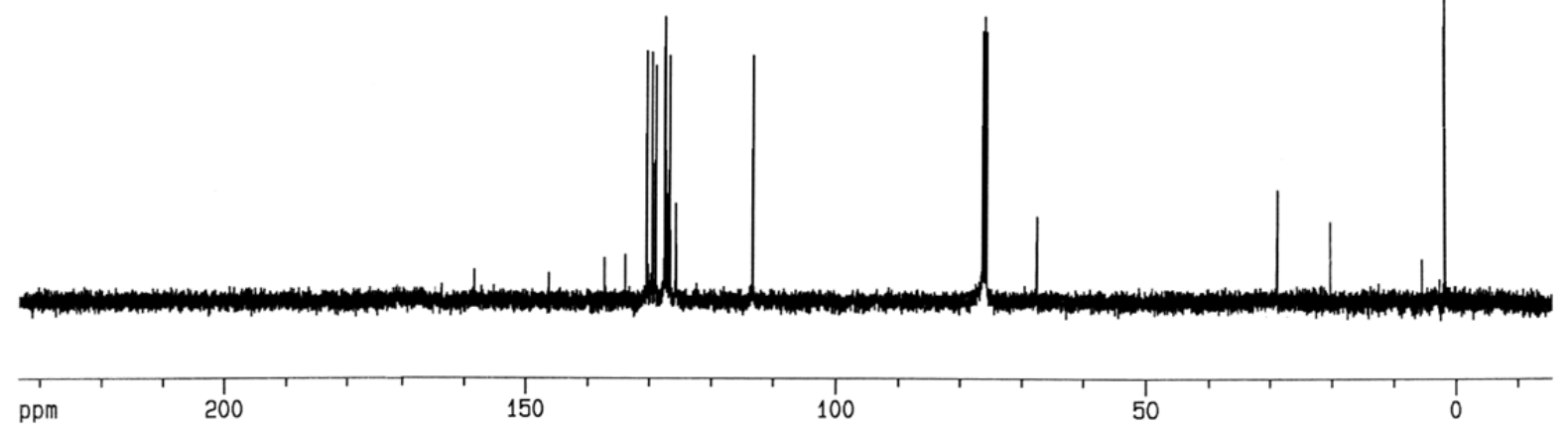

${ }^{13} \mathrm{C}$ NMR spectrum of 2-(4-(3,3,3-tris(trimethylsilyl)propoxy)phenyl)-4,5-diphenyl-1-p-tolyl-1H-imidazole (5b) in $\mathrm{CDCl}_{3}$ 


\section{2-(4-(4,4,4-tris(trimethylsilyl)butoxy)phenyl)-4,5-diphenyl-1-p-tolyl-1H-imidazole (6b)}

White powder (93\%), M.P. $=140-142{ }^{\circ} \mathrm{C}$, IR $\left(\mathrm{KBr}, \mathrm{cm}^{-1}\right)=3059,2954,1616,1511,1472,1253,1176,1027,898$, 836, 698. ${ }^{1} \mathrm{H}$ NMR $\left(\mathrm{CDCl}_{3}, 400 \mathrm{MHz}\right): \delta_{\mathrm{H}} 0.13$ (s, 27H, $\left.\mathrm{SiMe}_{3}\right), 1.76\left(\mathrm{t}, J=3.9 \mathrm{~Hz}, 2 \mathrm{H}, \mathrm{CH}_{2}\right), 1.93-2.01$ (m, 2H, $\left.\mathrm{CH}_{2}\right), 2.31\left(\mathrm{~s}, 3 \mathrm{H}, \mathrm{CH}_{3}\right), 3.87\left(\mathrm{t}, J=6.3 \mathrm{~Hz}, 2 \mathrm{H}, \mathrm{CH}_{2}\right), 6.76(\mathrm{~d}, J=8.7 \mathrm{~Hz}, 2 \mathrm{H}, \mathrm{Ar}-\mathrm{H}), 6.91(\mathrm{~d}, J=8.2 \mathrm{~Hz}, 2 \mathrm{H}, \mathrm{Ar}-$ H), 7.04 (d, J=8.2 Hz, 2H, Ar-H), 7.11-7.30 (m, 8H, Ar-H), 7.35 (d, J=8.7 Hz, 2H, Ar-H), 7.58 (d, J= 7.3 Hz, 2H, Ar-H). ${ }^{13} \mathrm{C} \mathrm{NMR}\left(\mathrm{CDCl}_{3}, 100 \mathrm{MHz}\right): \delta_{\mathrm{C}} 1.72\left(\mathrm{SiMe}_{3}\right), 4.43\left(\mathrm{C}\left(\mathrm{SiMe}_{3}\right)_{3}\right), 20.17\left(\mathrm{CH}_{3}\right), 26.10,28.70,67.56\left(\mathrm{CH}_{2}\right)$, $113.02,125.42,126.39,126.74,127.09,127.14,127.25,128.65,129.25,129.86,130.12,133.59,136.99,145.98$, 157.98. Anal.Calc. for $\mathrm{C}_{41} \mathrm{H}_{54} \mathrm{~N}_{2} \mathrm{OSi}_{3}$ : C, 72.94; H, 8.06; N, 4.15\%. Found: C, 72.68; H, 7.94; N, 4.21\%. 

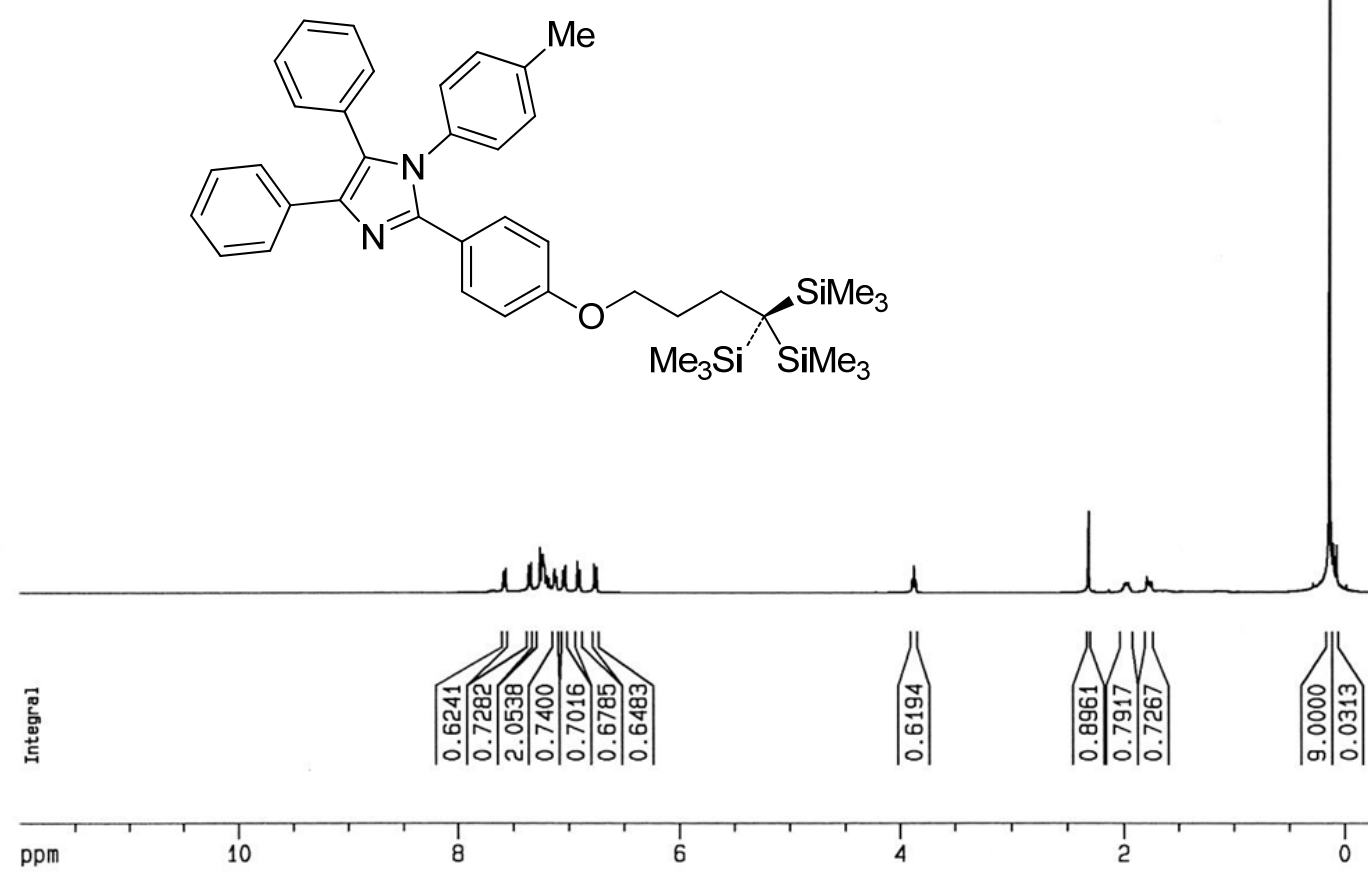

${ }^{1} \mathrm{H}$ NMR spectrum of 2-(4-(4,4,4-tris(trimethylsilyl)butoxy)phenyl)-4,5-diphenyl-1-p-tolyl-1H-imidazole (6b) in $\mathrm{CDCl}_{3}$ 
<smiles>COCCCC([SiH3])([SiH3])[SiH2]C</smiles>

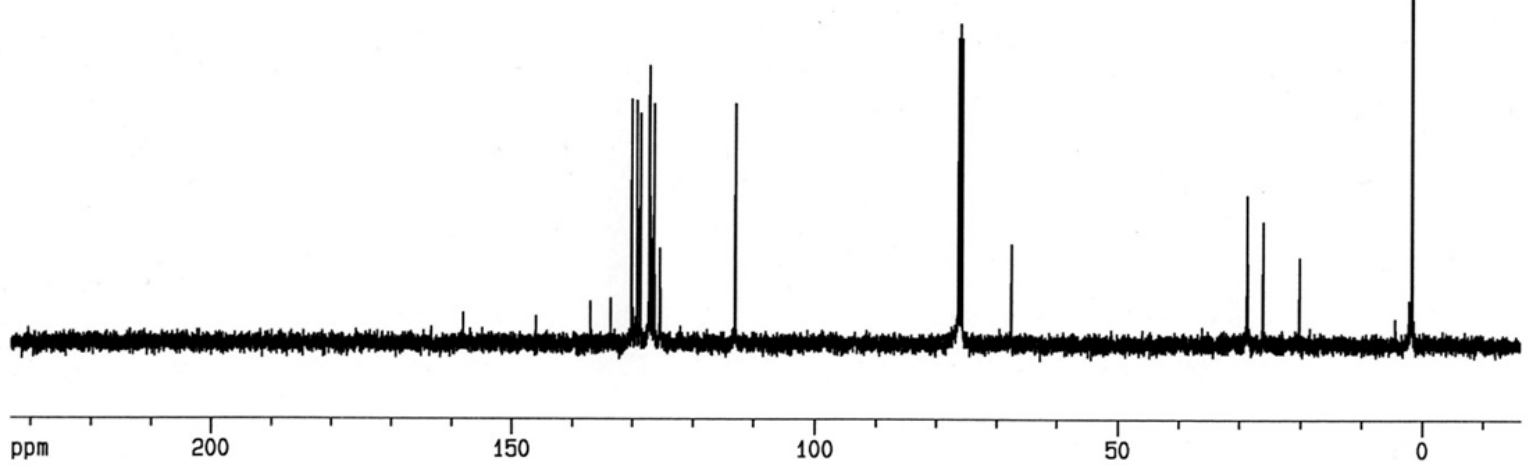

${ }^{13} \mathrm{C}$ NMR spectrum of 2-(4-(4,4,4-tris(trimethylsilyl)butoxy)phenyl)-4,5-diphenyl-1-p-tolyl-1H-imidazole (6b) in $\mathrm{CDCl}_{3}$ 


\section{2-(4-(5,5,5-tris(trimethylsilyl)pentyloxy)phenyl)-4,5-diphenyl-1-p-tolyl-1H-imidazole (7b)}

White powder $(92 \%)$, M.P. $=202-204^{\circ} \mathrm{C}, \mathrm{IR}\left(\mathrm{KBr}, \mathrm{cm}^{-1}\right)=3057,2951,1608,1577,1514,1476,1254,1174,1030$, 840, 696. ${ }^{1} \mathrm{H}$ NMR $\left(\mathrm{CDCl}_{3}, 400 \mathrm{MHz}\right): \delta_{\mathrm{H}} 0.17\left(\mathrm{~s}, 27 \mathrm{H}, \mathrm{SiMe}_{3}\right), 1.72-1.78\left(\mathrm{~m}, 6 \mathrm{H}, \mathrm{CH}_{2}\right), 2.36\left(\mathrm{~s}, 3 \mathrm{H}, \mathrm{CH}_{3}\right), 3.97$ (t, $\left.J=5.9 \mathrm{~Hz}, 2 \mathrm{H}, \mathrm{CH}_{2}\right), 6.80(\mathrm{~d}, J=8.9 \mathrm{~Hz}, 2 \mathrm{H}, \mathrm{Ar}-\mathrm{H}), 6.96(\mathrm{~d}, J=8.2 \mathrm{~Hz}, 2 \mathrm{H}, \mathrm{Ar}-\mathrm{H}), 7.09$ (d, $\left.J=8.1 \mathrm{~Hz}, 2 \mathrm{H}, \mathrm{Ar}-\mathrm{H}\right)$, 7.16-7.31 (m, 8H, Ar-H), $7.40(\mathrm{~d}, J=8.8 \mathrm{~Hz}, 2 \mathrm{H}, \mathrm{Ar}-\mathrm{H}), 7.63(\mathrm{~d}, J=7.1 \mathrm{~Hz}, 2 \mathrm{H}, \mathrm{Ar}-\mathrm{H}) .{ }^{13} \mathrm{C}$ NMR $\left(\mathrm{CDCl}_{3}, 100\right.$ MHz): $\delta_{\mathrm{C}} 1.72\left(\mathrm{SiMe}_{3}\right), 4.38\left(\mathrm{C}\left(\mathrm{SiMe}_{3}\right)_{3}\right), 20.16\left(\mathrm{CH}_{3}\right), 25.50,29.60,29.83,66.29\left(\mathrm{CH}_{2}\right), 112.99,121.99,125.39$, $126.35,126.71,127.06,127.10,127.23,128.62,129.21$, 129.84, 130.09, 133.55, 133.58, 136.94, 157.92. Anal.Calc. for $\mathrm{C}_{42} \mathrm{H}_{56} \mathrm{~N}_{2} \mathrm{OSi}_{3}:$ C, 73.20; H, 8.19; N, 4.06\%. Found: C, 73.09; H, 8.23; N, 4.11\%. 
言
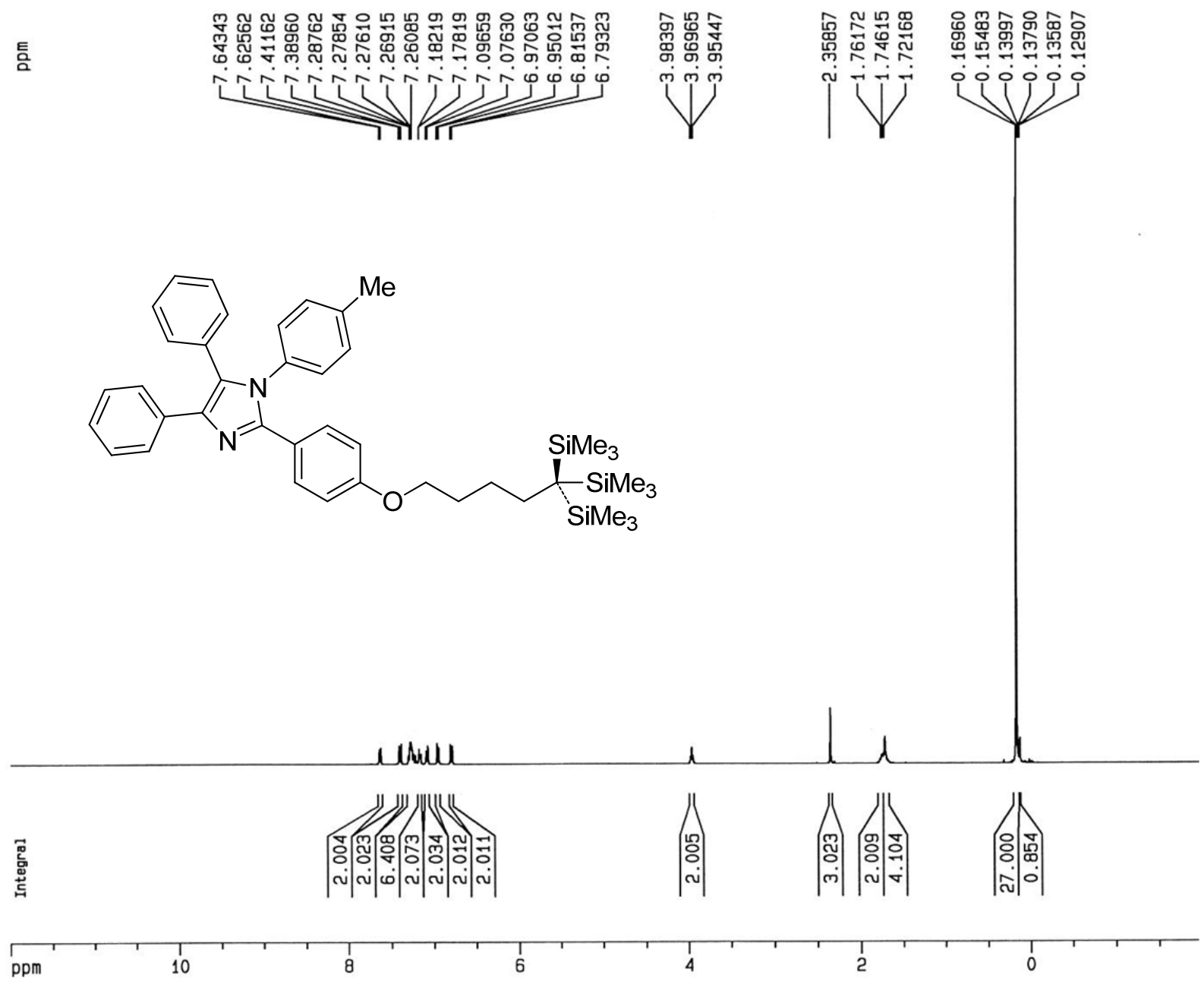

${ }^{1} \mathrm{H}$ NMR spectrum of 2-(4-(5,5,5-tris(trimethylsilyl)pentyloxy)phenyl)-4,5-diphenyl-1-p-tolyl-1H-imidazole (7b) in $\mathrm{CDCl}_{3}$ 

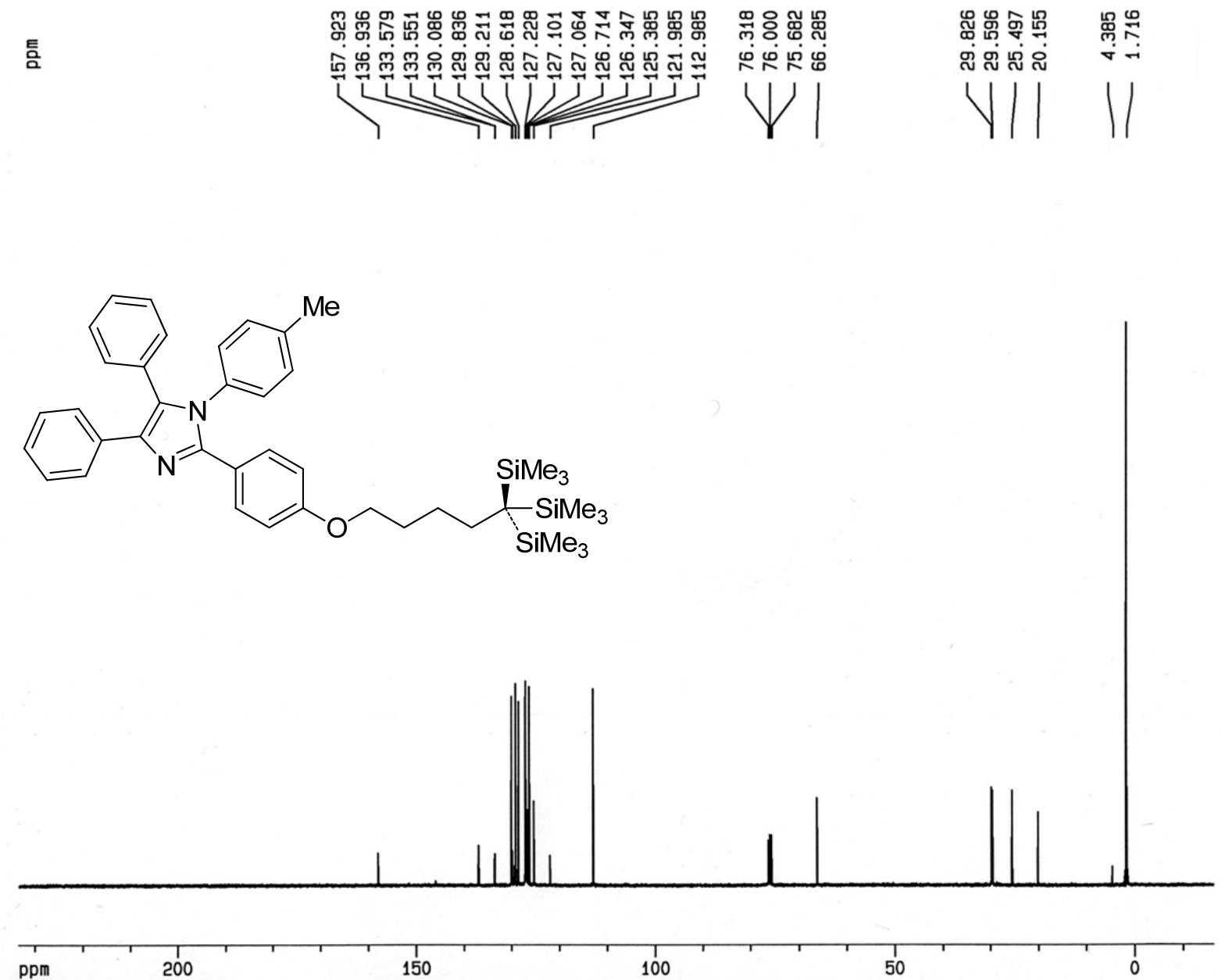

${ }^{13} \mathrm{C}$ NMR spectrum of 2-(4-(5,5,5-tris(trimethylsilyl)pentyloxy)phenyl)-4,5-diphenyl-1-p-tolyl-1 $H$-imidazole (7b) in $\mathrm{CDCl}_{3}$ 


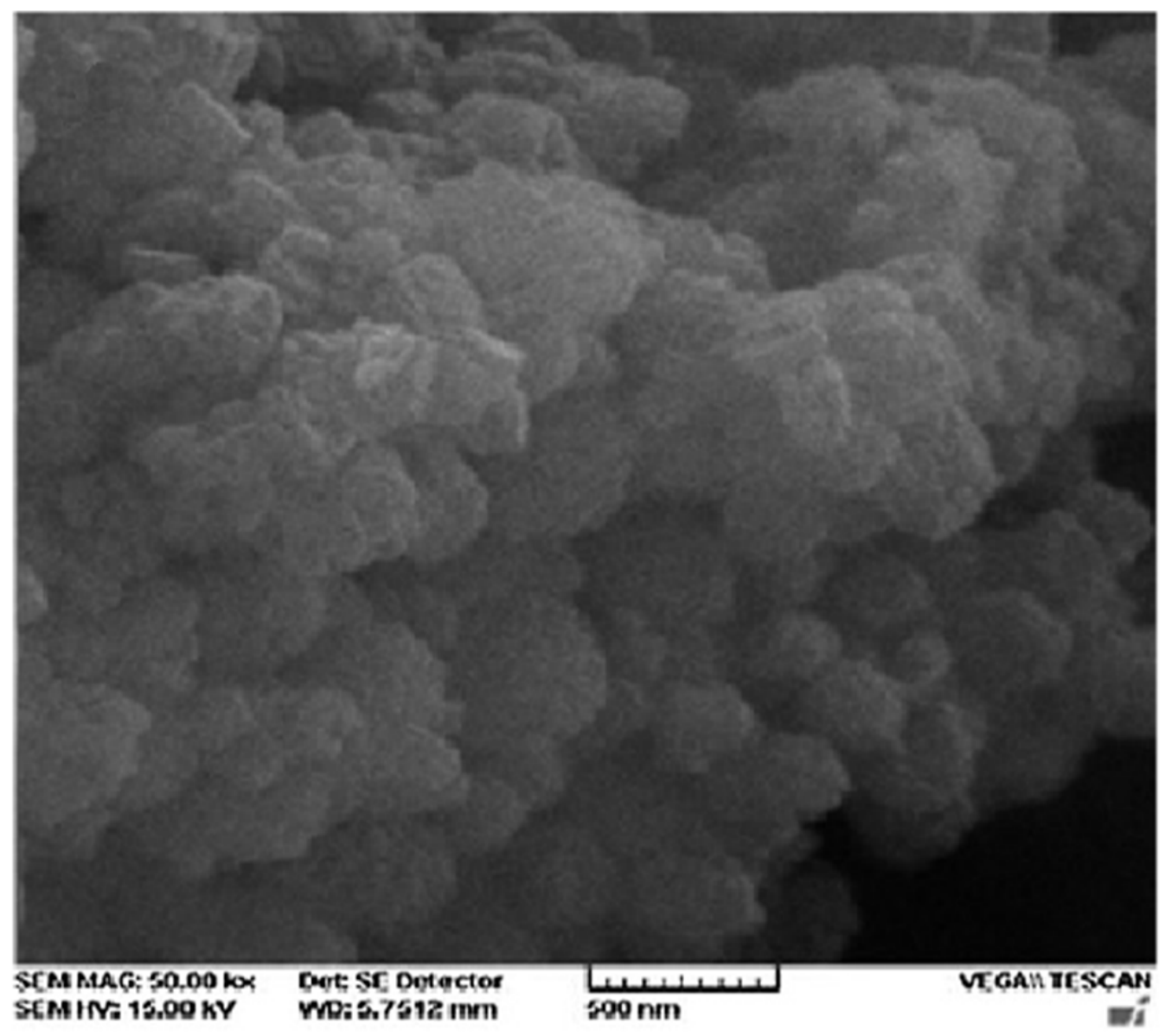

S1. SEM image of H-ZSM-5 


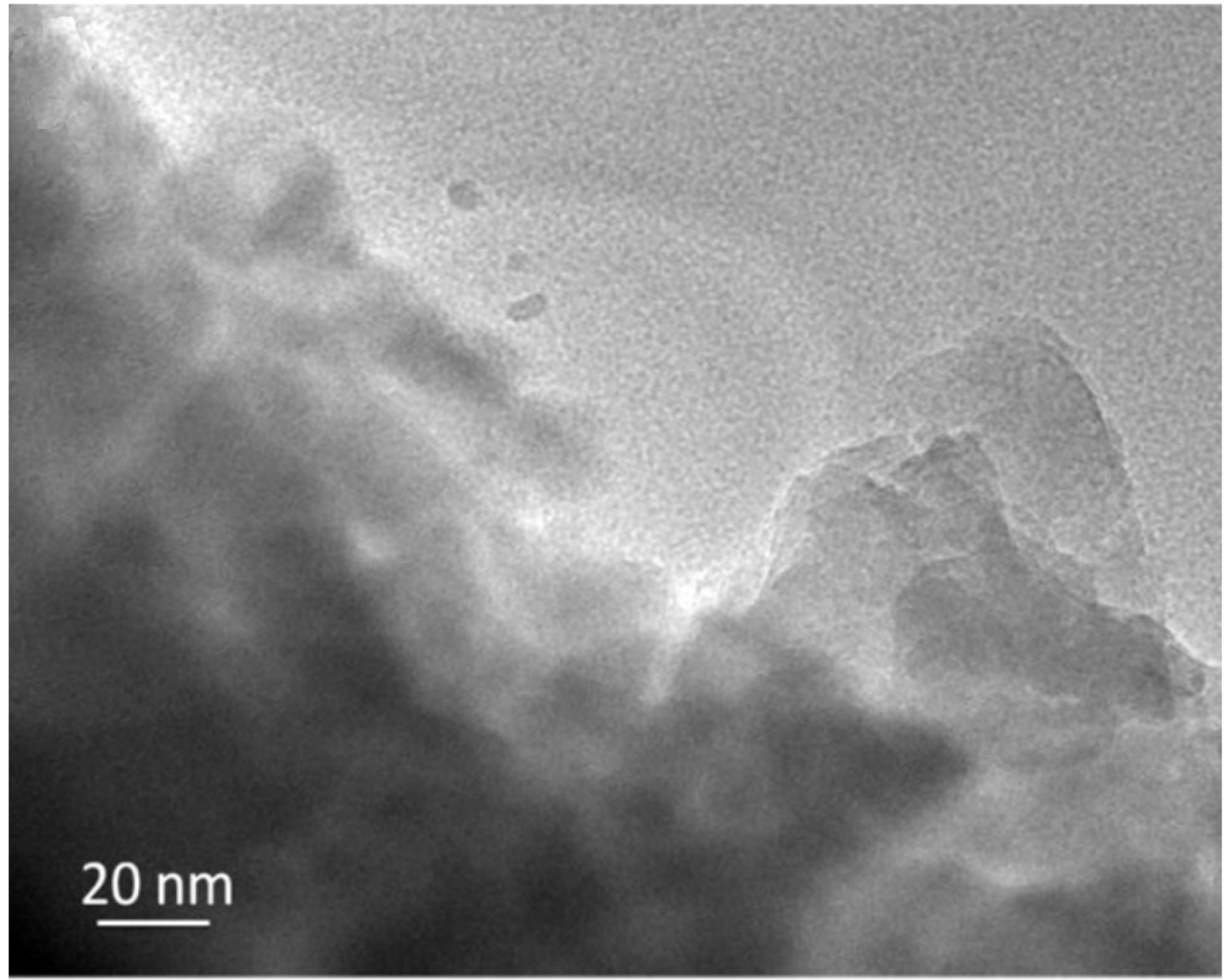

S2.TEM image of $\mathrm{H}-\mathrm{ZSM}-5$ 


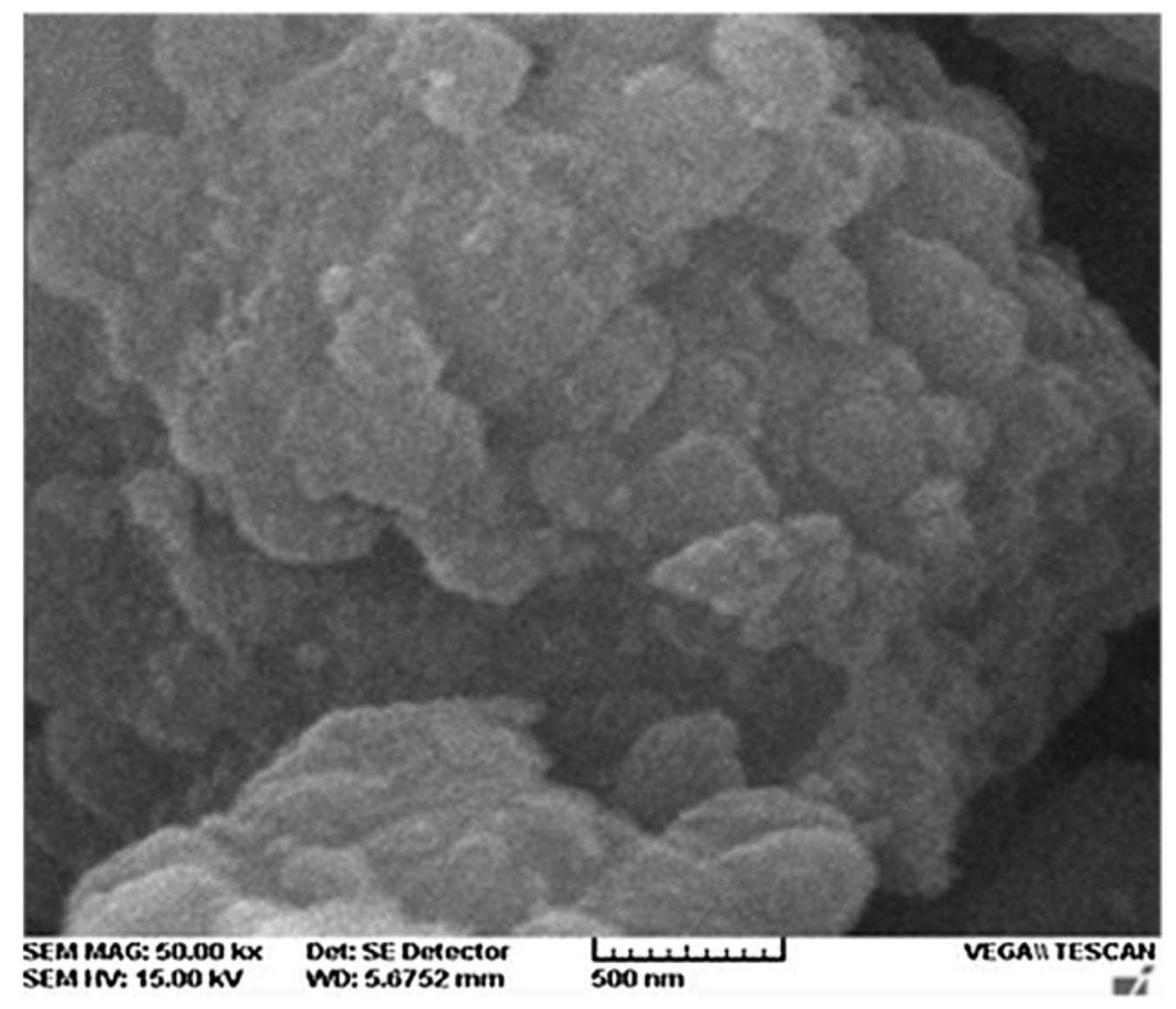

S3. SEM image of Cu/ZSM-5 


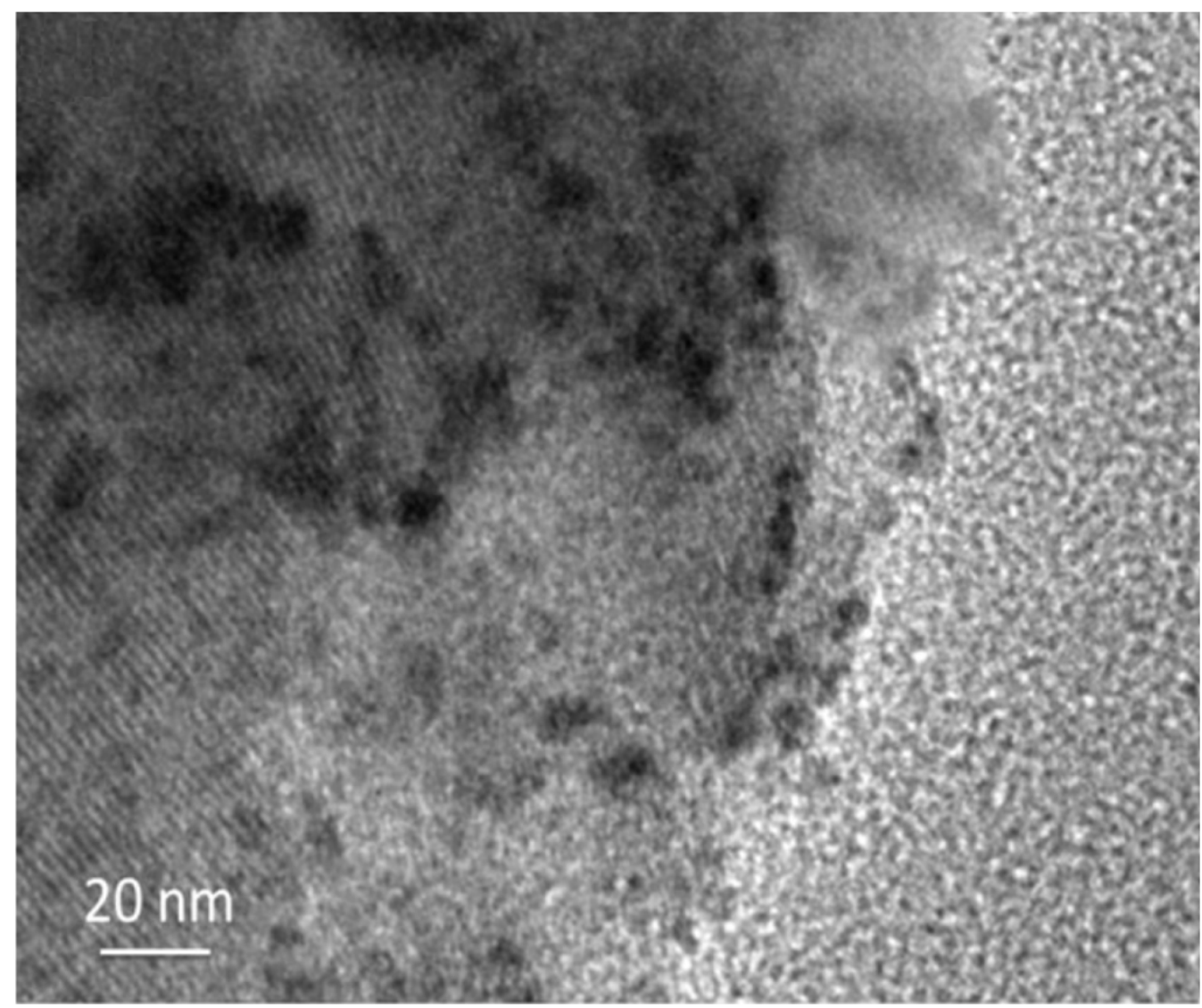

S4. TEM image of Cu /ZSM-5 


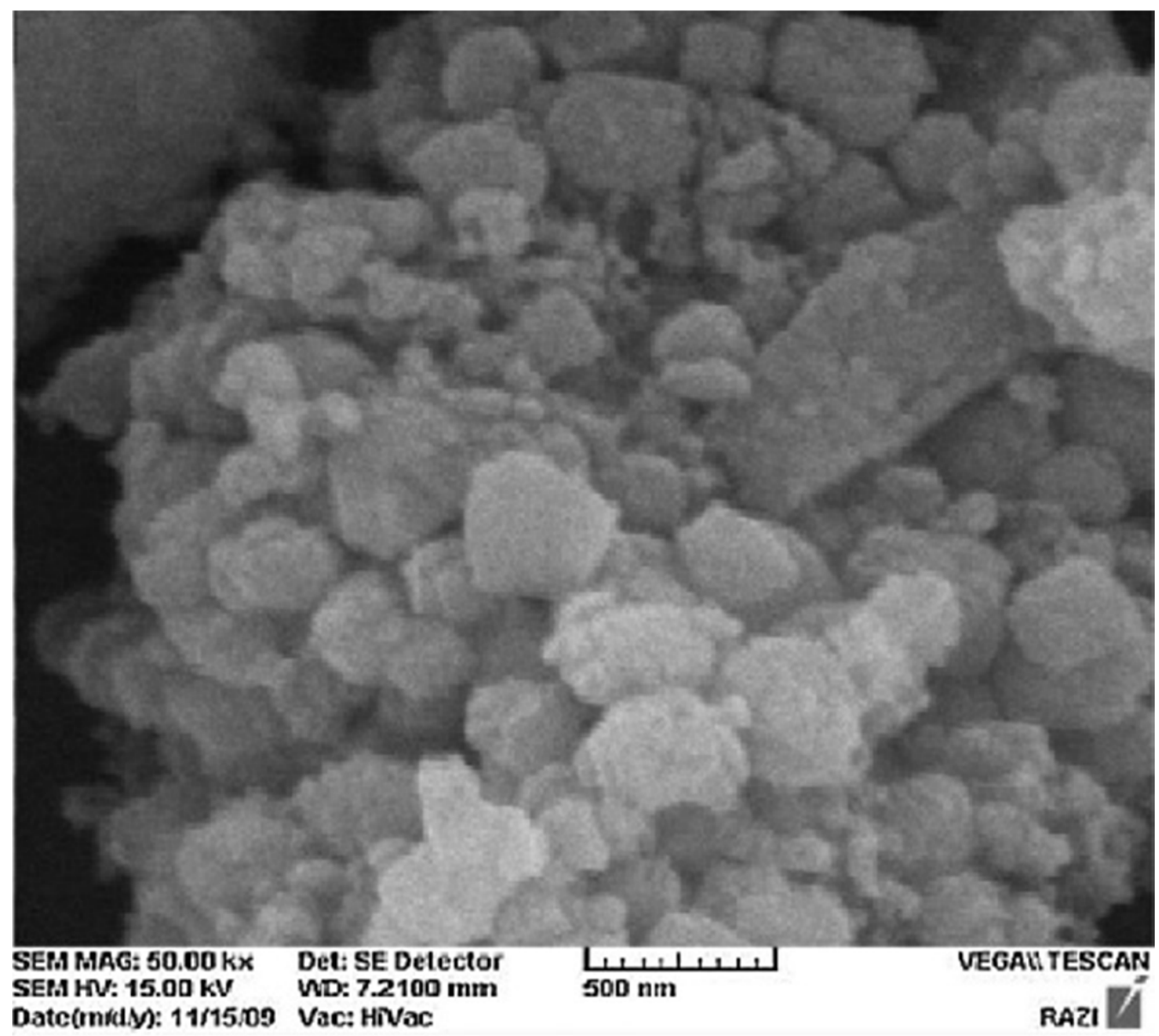

S5. SEM image of Cu-Fe/ZSM-5 


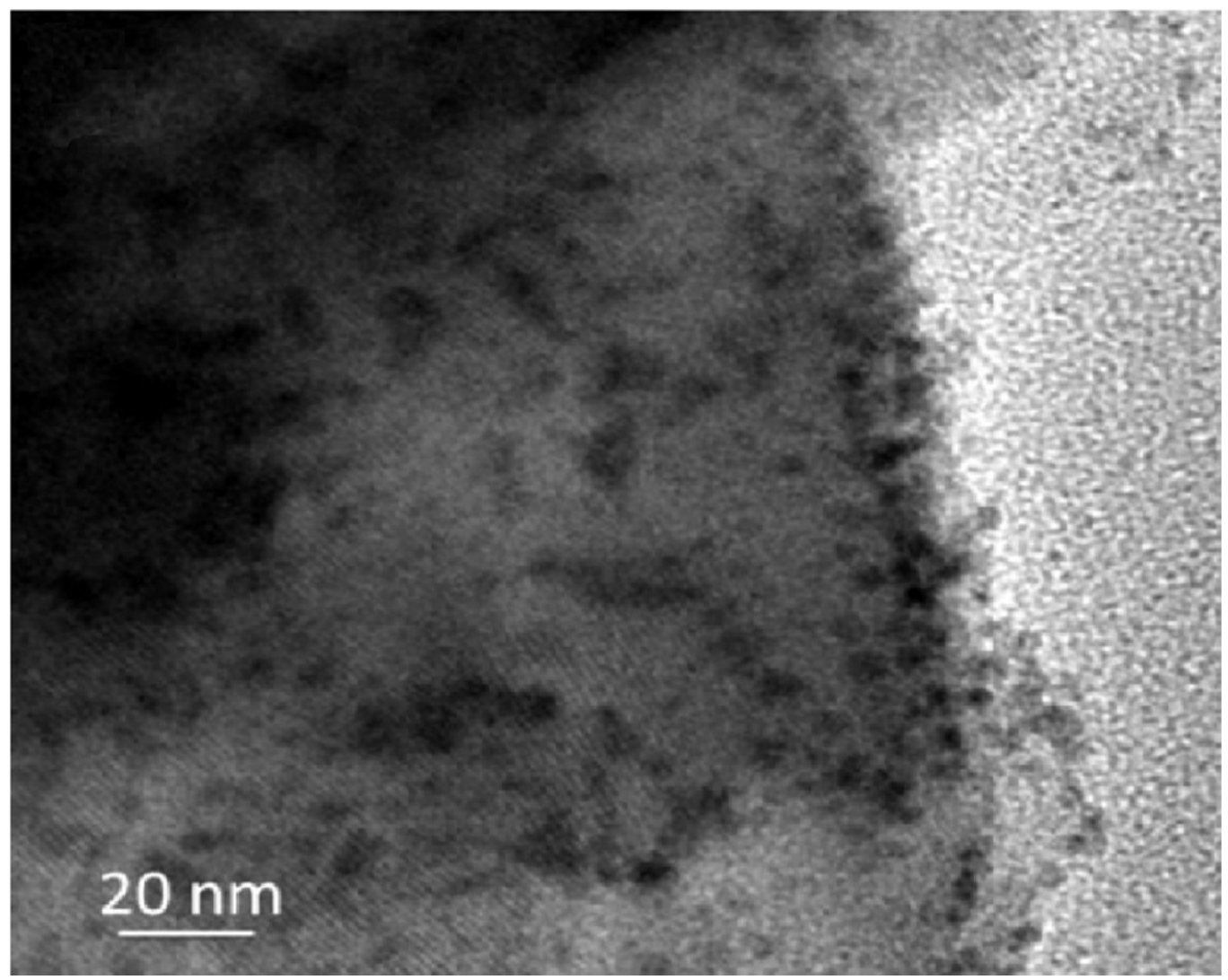

S6. TEM image of Fe-Cu/ZSM-5 


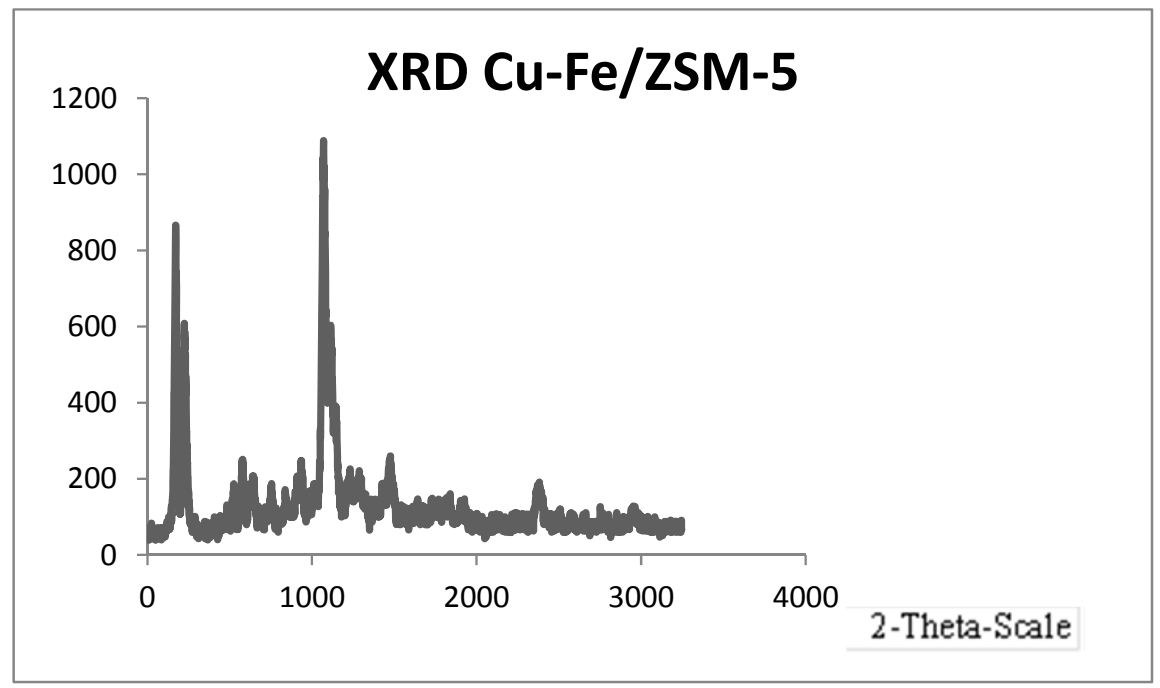

S7. The XRD patterns of Fe-Cu/ZSM-5 